\title{
RANDOM WALKS AND A SOJOURN DENSITY PROCESS OF BROWNIAN MOTION(1)
}

\author{
BY \\ F. B. KNIGHT
}

The sojourn times for the Brownian motion process in 1 dimension have often been investigated by considering the distribution of the length of time spent in a fixed set during a fixed time interval. In this approach the sojourn time is either treated as a functional leading to a differential equation (as in $[9, \mathrm{Kac}]$ ) or else the method of moments is used (as in [3, Darling and Kac] in a more general situation). An alternative approach, however, is suggested by a theorem of $\mathbf{H}$. Trotter [14] that as a set function, the sojourn time up to a time $t$ is a. s. absolutely continuous with respect to Lebesgue measure, and that, in fact, it has a density function $f(x, t, w)$ which is continuous in $(x, t)$. This theorem creates the possibility of considering the sojourn density function as a stochastic process with parameter $x$, coexistent with the Brownian motion in $t$ from which it is defined. The study of sojourn times then becomes equivalent to the study of this new process.

The possible advantage of such an approach depends on the fact that when this process is considered in a suitable random time interval it proves to be very amenable to investigation. If one sets, for example, $T=\inf _{t}: f(0, t, w)>\alpha$, then $f(x, T, w)$ turns out to be the diffusion process with $x \geqq 0$ as parameter, initial value $\alpha$, infinitesimal generator $4 y\left(d^{2} / d y^{2}\right)$, and an absorbing barrier at 0 . This fact illustrates our main result, which is contained below in Theorem 2.2. Its proof, together with a new proof of the theorem of [14], is accomplished by using a strong approximation to Brownian motion by means of the classical random walks (i.e., "coin tossing') which appeared in [10]. It seems of methodological interest that special properties of this construction are used essentially, so that although the result concerns only Brownian motion there is apparently no easy way to avoid using discrete random walks in the proof $\left({ }^{2}\right)$.

The first part of the paper introduces the random walk versions of the limiting sojourn density processes and includes a weak limit of their joint distributions,

Received by the editors September 14, 1962.

(1) This work was supported by the Air Force Office of Scientific Research.

(2) After the completion of this work, the author learned of the recent but prior paper [13] of D. Ray, which contains a proof of Theorem 2.2 carried out briefly (and elegantly) by analytical methods. It thus emerges that the random walks are not required. Even according to, [13]. however, they provide useful motivation for the theorem. 
taken in order to derive the joint distributions of the limit process. Since there is no existence proof for the limit process at this stage (except that of [14]) and since in any case it is not obvious what the implications of the random walk limit distributions for the limit process are, the limit distributions have at first only a formal significance for Brownian motion. In the second part of the paper it is shown that these limit distributions belong to a sojourn density process of Brownian motion. Only at this stage of the argument is the strong approximation of [10] required. This sojourn process is then used, along with a family of related processes, to investigate the sojourn time density under the conditions present in [14]. There follows a new proof of the theorem of [14] and a derivation of some estimates concerning it. The main problem considered is that of estimating the distribution of the maximum in $x$ of the sojourn density up to a fixed time $t$. Whereas in [14] no estimate for this distribution could be derived because of the neglect of the higher dimensional joint distributions which was required by the method itself, the present method is not limited in this way. It raises other problems, however, and the estimate which we give is only approximate. Using this estimate it is proved that the sojourn density process is a limit process of the random walk sojourn processes in the same sense (uniformly in finite time intervals a.s.) that Brownian motion is a strong limit of the random walks. This facilitates the construction, which was indicated in Remark 3 of [10], of general 1-dimensional diffusion processes (the processes of Feller) by means of the random walk approximation, and consequently, we present anew this construction. In the final paragraphs are mentioned the sojourn density processes defined on these general diffusion processes.

1. Let $R(n), n \geqq 0, R(0)=0$, be the classical random walk on the integers, with transition function $p(k, k+1)=p(k, k-1)=\frac{1}{2}$ (a more complete definition is given, for example, in [10]). In order to escape the necessity of referring repeatedly to certain exceptional sets of probability 0 , we shall assume also that for each pair $k_{1}, k_{2}$ there are infinitely many disjoint intervals $(m, n)$ for which $R(m)=k_{1}$ and $R(n)=k_{2}$. Since the set where this holds is of probability 1 , as is easily seen, its complement may be excluded from the basic probability space without altering the joint distributions of $R(n)$. The random variables of the sojourn process require a special notation which is now introduced.

Definition 1.1. For each $k>0$ and $m \geqq 0$, let $S(m, k)$ be the number of integers $n>0$ such that $R(n-1)=k$ and $R(n)=k+1$, and such that there are at most $m$ values of $i, 0<i \leqq n$, for which $R(i-1)=0$ and $R(i)=1$. Stated in words, $S(m, k)$ is the number of steps of $R(n)$ from $k$ to $k+1$ up to the $(m+1)$ th step from 0 to 1 . Lastly, let $S(m, 0)=m$.

In the remainder of this paper we shall usually give definitions and proofs in the second style only, using such terms as "steps" and "passages" from $a$ to $b$, to avoid excessive formality when no ambiguity can arise. There follows 
a key theorem. The statement is quite inescapable intuitively, but the proof is unexpectedly complex.

THEOREM 1.1. For each $m \geqq 0, S(m, k), k \geqq 0$, is a homogeneous Markov chain on the non-negative integers with $S(m, 0)=m$ and 1-step transition function

$$
p\left(i_{1}, i_{2}\right)=(-1)^{i_{2}}\left(\begin{array}{r}
-i_{1} \\
i_{2}
\end{array}\right) 2^{-\left(i_{1}+i_{2}\right)} .
$$

Proof. For $k \geqq 0$ fixed, let $j_{1, i}, 1 \leqq i \leqq i_{1}$, be the least integers such that $R(n)$ makes exactly $i$ steps from $k$ to $k+1$ for $0 \leqq n \leqq j_{1, i}$. Thus in particular $R\left(j_{1, i}-1\right)=k$ and $R\left(j_{1, i}\right)=k+1$. Let also $j_{2, i}, 1 \leqq i \leqq i_{1}$, be the least integers greater than $j_{1, i}$, respectively, such that $R\left(j_{2, i}\right)=k$. It is easily seen that both $j_{1, i}$ and $j_{2, i}$ are stopping times for $R(n)$. Next, let $F(i), 1 \leqq i \leqq i_{1}$, be the field generated by both $R(n), 0 \leqq n \leqq j_{1, i}$ (i.e., all measurable sets $S$ such that $S \cap\left\{j_{1, i} \leqq r\right\}$ is in the field generated by $R(n), 0 \leqq n \leqq r$, for all $r \geqq 0$, the "past of $R(n)$ up to time $j_{1, i}$ '") and jointly, $R\left(j_{2, i}+n\right), 0 \leqq n<\infty$. It is clear that $S\left(m, k_{1}\right)$, for $0 \leqq k_{1} \leqq k$, is measurable over $F(i)$ for each $i$. Finally, let $U_{k_{2}, i}$, $k<k_{2}$, be the number of steps of $R(n)$ from $k_{2}$ to $k_{2}+1$ for $j_{1, i} \leqq n<j_{2, i}$, and let $G(i), 1 \leqq i \leqq i_{1}$, be the field generated by $R\left(j_{i, i}+n\right), 0 \leqq n \leqq j_{2, i}-j_{1, i}$ (the "past of $R\left(j_{1, i}+n\right), n \geqq 0$, up to time $j_{2, i}-j_{1, i}$ "). Clearly $U_{k_{2}, i}$ is measurable over $G(i)$, while $G(i)$ and $F(i)$ are independent. For $j \neq i, 1 \leqq j \leqq i_{1}, U_{k_{2}, j}$ is also measurable over $F(i)$, from which it follows that $U_{k_{2}, i}, 1 \leqq i \leqq i_{1}$, are jointly independent of each other and of the field $F$ defined by $F=\bigcap_{i=1}^{i_{1}} F(i)$. Also, $S\left(m, k_{1}\right)$ is measurable over $F$, while under the condition that $S(m, k)=i_{1}$ (which we are going to take as given) we have $S\left(m, k_{2}\right)=\sum_{i=1}^{i_{1}} U_{k_{2}, i}$. Since $\left\{S(m, k)=i_{1}\right\}$ is in $F$, it follows that, given $S(m, k)=i_{1}, S\left(m, k_{1}\right)$ and $S\left(m, k_{2}\right)$ are conditionally independent. Therefore, $S(m, k), k \geqq 0$, is a Markov chain.

To find the transition probabilities it is sufficient to determine the distribution of the number of steps from $k+1$ to $k+2$ for $j_{1,1} \leqq n<j_{2,1}$, since the above reasoning shows that the conditional distribution of $S(m, k+1)$ given $\left\{S(m, k)=i_{1}\right\}$ is that of the sum of $i_{1}$ independent and identically distributed random variables equalling the numbers of steps from $k+1$ to $k+2$ in the corresponding intervals $j_{1, i} \leqq n<j_{2, i}, 1 \leqq i \leqq i_{1}$. Since $R\left(i_{1,1}\right)=k+1$, the required distribution is that of the number of steps of $R(n)$ from $k+1$ to $k+2$, starting at $k+1$, before reaching $k$, and this is a geometric distribution with $p=\frac{1}{2}$. The corresponding generating function is $(2-s)^{-1}$, and $p\left(i_{1}, i_{2}\right)$ is therefore the coefficient of $s^{i_{2}}$ in $(2-s)^{-i_{1}}$. Expansion using the binomial theorem yields the coefficients easily, completing the proof.

The proof of this theorem extends to certain other processes related to $S(m, k)$ which are to be used in what follows. Accordingly, we extend Definition 1.1 to a further parameter $b$, taking for convenience $b \leqq 0$. The new definition reduces to that of $S(m, k)$ when $b=0$. 
DEFINITION 1.2. Let $S(b, m, k)$, for integer values of the arguments and $b \leqq 0, m \geqq 0, k>0$, be the number of steps of $R(n)$ from $b+k$ to $b+k+1$ up to the $(m+1)$ th step from $b$ to $b+1$, and let $S(b, m, 0)=m$.

By Theorem 1.1 the numbers of steps from $b+k$ to $b+k+1, k \geqq 0$, following the first arrival at $b$ and up to the $(m+1)$ th step from $b$ to $b+1$ define a Markov chain with initial value $m$. If it is shown that the numbers of steps from $b+k$ to $b+k+1$ before the first arrival at $b$ also define a Markov chain, independent of the preceding one, it follows that $S(b, m, k)$ is a Markov chain, and its transition function can be formed from those of its components. In this way one is led to a corollary of Theorem 1.1.

Corollary 1.1. $S(b, m, k), k \geqq 0$, is a Markov chain with 1-step transition function given by

$$
\begin{aligned}
p\left(k ; i_{1}, i_{2}\right)= & P\left(S(b, k+1, m)=i_{2} \mid S(b, k, m)=i_{1}\right) \\
& = \begin{cases}(-1)^{i_{2}}\left(\begin{array}{c}
-\left(i_{1}+1\right) \\
i_{2}
\end{array}\right) 2^{-\left(i_{1}+i_{2}+1\right)} & \text { for } 0 \leqq k<-b \\
(-1)^{i_{2}}\left(\begin{array}{c}
-i_{1} \\
i_{2}
\end{array}\right) 2^{-\left(i_{1}+i_{2}\right)} & \text { for }-b \leqq k .\end{cases}
\end{aligned}
$$

Proof. For $-b \leqq k$ the proof of Theorem 1.1 applies directly to the present situation. The only difference is that $S(b, m,-b)$ replaces the initial value $m$ of Theorem 1.1. For $0 \leqq k<-b_{2}$ one has to introduce an additional stopping time $j_{2,0}<j_{1,1}$, equal to the index of the first passage to $b+k$, and to include in $S\left(b, m, k_{2}\right)$ the number of steps of $R(n)$ from $b+k_{2}$ to $b+k_{2}+1$ for $0 \leqq n \leqq j_{2,0}$ as well as those following $j_{1,1}$. If $k_{2}=k+1$, this addition is another geometric random variable independent of $R\left(j_{1,1}+n\right), n \geqq 0$, which implies that the transition function $p\left(k ; i_{1}, i_{2}\right)$ is in this case the convolution of $i_{1}+1$ geometric random variables. The corollary is proved.

The next task is to compute the $n$-step transition function of $S(m, k)$ and then, after introducing the appropriate scale change, let $n$ become large to derive the transition function and joint distributions of a continuous parameter limit process.

THEOREM 1.2. Let $p^{(n)}\left(i_{1}, i_{2}\right)$ be the $n$-step transition function corresponding to $p\left(i_{1}, i_{2}\right)$, and let $p^{([n x])}([n \alpha],[n \beta])=f_{n}(x ; \alpha, \beta), x \geqq 0, \alpha \geqq 0$, where $[y]$ is the largest integer not exceeding $y$. Then for $\beta>0$,

$$
\begin{aligned}
\lim _{\rightarrow \infty} n f_{n}(x ; \alpha, \beta) & =\frac{\alpha}{x^{2}} \exp \left(-\frac{\alpha+\beta}{x}\right) \sum_{k=0}^{\infty} \frac{1}{k !} \frac{1}{(k+1) !}\left(\frac{\sqrt{ }(\alpha \beta)}{x}\right)^{2 k} \\
& =\left(\frac{\exp \left(-\frac{\alpha+\beta}{x}\right)}{i x}\right) \sqrt{\frac{\alpha}{\beta}} J_{1}\left(\frac{2 i}{x} \sqrt{ }(\alpha \beta)\right)
\end{aligned}
$$


where $J_{1}$ is the Bessel function of the first kind, and $\lim _{n \rightarrow \infty} f_{n}(x ; \alpha, 0)=e^{-x / x}$. Denoting the former limit by $f(x ; \alpha, \beta)$, and the latter by $F(x ; \alpha, 0)$, and for $\beta \geqq 0$ setting $F(x ; \alpha, \beta)=F(x ; \alpha, 0)+\int_{0}^{\beta} f(x ; \alpha, \gamma) d \gamma$, the corresponding joint distributions converge to those of $F(x ; \alpha, \beta)$. More precisely, for all $0<x_{1}<\cdots<x_{k}$ and intervals $\left[\beta_{1,1}, \beta_{2,1}\right), \cdots,\left[\beta_{1, k}, \beta_{2, k}\right), \beta_{1, i} \leqq \beta_{2, i}, 1 \leqq i \leqq k$, we have

$$
\begin{gathered}
\lim _{n \rightarrow \infty} \sum_{\left(i_{2}, k / n\right) \in\left[\beta_{1, k}, \beta_{2}, k\right)} \cdots \sum_{\left(i_{2}, 1 / n\right) \in\left[\beta_{1}, 1, \beta_{2}, 1\right)} f_{n}\left(x_{1} ; \alpha, i_{(2,1) / n}\right) \\
\cdot f_{n}\left(x_{2}-x_{1} ; i_{(2,1) / n}, i_{(2,2) / n}\right) \cdots f_{n}\left(x_{k}-x_{k-1} ; i_{(2, k-1) / n}, i_{(2, k) / n}\right) \\
=\int_{\left[\beta_{1, k}, \beta_{2, k}\right)} \cdots \int_{\left[\beta_{1}, 1, \beta_{2}, 1\right)} F\left(x_{1} ; \alpha, d \beta_{1}\right) F\left(x_{2}-x_{1} ; \beta_{1}, d \beta_{2}\right) \\
\cdots F\left(x_{k}-x_{k-1} ; \beta_{k-1}, d \beta_{k}\right) .
\end{gathered}
$$

Proof. It will be shown first that $p^{(n)}\left(1, i_{2}\right)$ has the generating function $G_{n}(s)=(n-(n-1) s) /(n+1-n s)$. Thus $G_{1}(s)=(2-s)^{-1}$, which we have already seen is correct for $n=1$. Assuming that $G_{n}(s)$ is correct for $n$ we compute the generating function for $n+1$ by observing that each of the $i_{2}$ steps resulting from $p^{(n)}\left(1, i_{2}\right)$ gives rise independently to a geometrically distributed number of steps contributing to $p^{(n+1)}(1, \cdot)$. This implies that the generating function is the composite function $G_{n+1}(s)=G_{n}\left((2-s)^{-1}\right)$, and the induction step follows immediately by substitution. It is easily seen that $p^{(n)}\left(i_{1}, i_{2}\right)$ is the $i_{1}$-fold convolution of $p^{(n)}\left(1, i_{2}\right)$, and hence is the coefficient of $s^{i_{2}}$ in $\left(G_{n}(s)\right)^{i_{1}}$. Rewriting $G_{n}(s)$ in the form

$$
\frac{1}{n(n+1)} \quad\left(\frac{1}{1-\frac{n}{n+1} s}\right)+\frac{n-1}{n}
$$

and expanding the $i_{1}$ th power by the binomial theorem it is seen that

$$
p^{(n)}\left(i_{1}, i_{2}\right)=\sum_{k=1}^{i_{1}}(-1)^{i_{2}}\left(\begin{array}{c}
i_{1} \\
k
\end{array}\right)\left(\begin{array}{c}
-k \\
i_{2}
\end{array}\right)(n-1)^{i_{1}-k} n^{i_{2}-i_{1}}(n+1)^{-\left(i_{2}+k\right)} .
$$

It follows that

$$
\begin{gathered}
f(x ; \alpha, \beta)=\lim _{n \rightarrow \infty} n f_{n}(x ; \alpha, \beta) \\
=\lim _{n \rightarrow \infty} n \sum_{k=1}^{[n \alpha]}(-1)^{[n \beta]}\left(\begin{array}{c}
{[n \alpha]} \\
k
\end{array}\right)\left(\begin{array}{c}
-k \\
{[n \beta]}
\end{array}\right)\left(1-\frac{1}{[n x]}\right)^{[n \alpha]}\left(1-\frac{1}{[n x]}\right)^{[n \beta]}\left([n x]^{2}-1\right)^{-k} \\
=\exp -\frac{\alpha+\beta}{x} \lim _{n \rightarrow \infty} n \sum_{k=1}^{[n \alpha]}(-1)^{[n \beta]}\left(\begin{array}{c}
{[n \alpha]} \\
k
\end{array}\right)\left(\begin{array}{c}
-k \\
{[n \beta]}
\end{array}\right)\left([n x]^{2}-1\right)^{-k} .
\end{gathered}
$$


It is not difficult to verify that the last expression converges term by term to that given in the theorem, and since the latter has as its integral $1-e^{-\alpha / x}$ the convergence of the entire sum will follow from the convergence to $e^{-\alpha / x}$ of the constant terms $f_{n}(x ; \alpha, 0)$. Going back to $G_{n}(s)$ it is seen that

$$
\lim _{n \rightarrow \infty} f_{n}(x ; \alpha, 0)=\lim _{n \rightarrow \infty}\left(\frac{[n x]}{[n x]+1}\right)^{[n \alpha]}=e^{-\alpha / x}
$$

and the convergence is uniform for $\alpha$ and $x$ bounded away from 0 . For application of these facts to show the convergence of the joint distributions, it is only necessary to observe that the term by term convergence is uniform for $\alpha, \beta$, and $x$ in finite intervals bounded away from 0 and for $k$ bounded from above. From this follows the convergence of the joint distributions for $\beta_{1, i}>0$ and $\beta_{2, i}<\infty$, and since the asserted limit is a probability measure it must be valid without these restrictions. The proof is therefore complete.

Theorem 1.2 shows that the processes $S(0, m, k), k \geqq 0$, when suitably normalized by setting $m=\left[n \alpha_{0}\right], k=[n x]$, and multiplying by $n^{-1}$, converge in the weak sense to a Markov process with homogeneous transition measure $F(x ; \alpha, d \beta)$, where $x \geqq 0$ denotes the parameter of the process, and $\alpha_{0}$ the initial value. We are going to extend this to the processes $S(b, m, k)$, but shall do so by means of the infinitesimal generators rather than the transition functions. It is therefore convenient to derive the infinitesimal generator of the process of Theorem 1.2 first, although it is known from [5] that this process is the diffusion process with generator $y d^{2} / d y^{2}$ and 0 as absorbing barrier. The proof then extends to the general case.

COROLlaRY 1.2. The transition measures $F(x ; \alpha, d \beta)$ correspond to the homogeneous Markov process (diffusion process) with infinitesimal generator $y d^{2} / d y^{2}$ operating in the space of continuous functions converging at $\infty$ and vanishing at 0 , which is an absorbing barrier.

Proof. In the derivation of Theorem 1.2 we introduced the change of scale $i_{1}=[n \alpha], i_{2}=[n \beta]$, which in terms of the generating functions means that we consider the function

$$
\left\{1-\frac{1}{[n x]}+\frac{1}{[n x]([n x]+1)}\left(\frac{1}{1-\frac{[n x] s}{[n x]+1}}\right)\right\}^{[n \alpha]},
$$

for which $n$ times the coefficient of $s^{i_{2}}$ is approximately $f(x ; \alpha, \beta)$. If this is replaced by a Laplace transform, by setting $s=e^{-\lambda / n}$, its limit as $n$ becomes large is therefore $\int_{0}^{\infty} e^{-\lambda \beta} F(x ; \alpha, d \beta)$. This limit is easily found to be $\exp -(\lambda \alpha / 1+\lambda x)$, a fact which may also be verified directly. Let $T_{x}$ be the semigroup of the process acting on bounded measurable functions on $[0, \infty)$, i.e., $T_{x} f(y)=\int_{0}^{\infty} f(\beta) F(x ; y, d \beta)$. 
Since, for $\lambda>0, T_{x}\left(e^{-\lambda y}\right)=\exp -(\lambda y / 1+\lambda x), T_{x}$ is strongly continuous on the set of functions $e^{-\lambda y}, \lambda \geqq 0$, in the uniform topology. It follows from the StoneWeierstrass theorem that the uniform linear closure of this set consists of all bounded continuous functions on $[0, \infty)$ converging at $\infty$ (i.e., continuous functions on $[0, \infty]$ ). Hence $T_{x}$ is strongly continuous on this space, and we can consider it as operating here. For fixed $\lambda$,

$$
\left.\frac{d}{d x} \exp \left(-\frac{\lambda y}{1+\lambda x}\right)\right]_{x=0}=\lambda^{2} y e^{-\lambda y}=y \frac{d^{2}}{d y^{2}} e^{-\lambda y}
$$

and the first term exists uniformly in $y \geqq 0$, which means that $e^{-\lambda y}$ is in the domain of the strong infinitesimal generator $A$ and that $A=y d^{2} / d y^{2}$ for $e^{-\lambda y}$. Since, obviously, $A(1)=0$, the generator has the same form for the functions $1-e^{-\lambda y}$. These functions generate the space of continuous functions vanishing at 0 and converging at $\infty$, for we have

$$
\left(1-e^{-\lambda_{1} y}\right)\left(1-e^{-\lambda_{2} y}\right)=\left(1-e^{-\lambda_{1} y}\right)+\left(1-e^{-\lambda_{2} y}\right)-\left(1-e^{-\left(\lambda_{1}+\lambda_{2}\right) y}\right)
$$

and the Stone-Weierstrass theorem may be applied. It follows by considering the identity $\lim _{\lambda \rightarrow \infty} \lambda R_{\lambda}(f)=f$, where $R_{\lambda}$ is the resolvent of the semigroup, that the range of $\lambda-A$ acting on the functions $1-e^{-\lambda_{1} y}$ likewise generates this space. Since the diffusion process of the corollary is completely determined by its resolvent acting on this range, the present process must be identical with that of the corollary.

Using the same method, we next will characterize the limit processes of $S(b, m, k)$ in terms of their infinitesimal generators and boundary conditions. The transition probabilities of these processes are deferred until following the proof of Theorem 2.2 .

Corollary 1.3. The joint distributions of $(1 / n)\left(S\left(\left[n x_{0}\right],\left[n \alpha_{0}\right],\left[n\left(x_{0}+x\right)\right]\right)\right)$, $x_{0} \leqq 0, \alpha_{0} \geqq 0, x>0$, converge to those of the inhomogeneous diffusion process with parameter $x \geqq 0$, initial value $\alpha_{0}$, and two disjoint intervals of homogeniety as follows. For $0 \leqq x<-x_{0}$ the generator is $y d^{2} / d y^{2}+d / d y$ and 0 is an entrance boundary; for $-x_{0} \leqq x$ the generator is $y d^{2} / d y^{2}$ and 0 is an exit boundary with the absorbing barrier boundary condition.

Note. By "diffusion" is understood, in particular, a process with path functions continuous in $x$, including at $x=-x_{0}$.

Proof. Let us suppose for the moment that it has been shown that the distributions at $x=-x_{0}$ converge. Then it follows directly as in the proof of Corollary 1.2 that for $x \geqq-x_{0}$ the process satisfies Corollary 1.3 with this limit distribution at $x=-x_{0}$ as "initial" distribution. The corollary will therefore be proved if it is shown that the limit distributions have the indicated form for $0<x<-x_{0}$, and that they define a process which is Markovian at $x=-x_{0}$. In this interval, the generator still contains the term $y d^{2} / d y^{2}$ corresponding to the contribution of the approximating random walks following their first arrivals at the (approximate) 
"starting point" $\left[n\left(x_{0}+x\right)\right] / n$ of the parameter at which the derivative of the semigroup is computed (in particular, near $x=0$ ). To this term must be added the contribution preceding the first arrival. Since this does not contribute to the initial value at $\left[n\left(x_{0}\right)\right] / n$ we see as before that the initial value at $x=0$ is $\alpha_{0}$. Let it now be assumed that $x=k / n<-x_{0}$, and consider the generating function of the number of steps of $R(n)$ from $\left[n\left(x_{0}+k / n\right)\right]$ to $\left[n\left(x_{0}+(k+1) / n\right)\right]$ before the first arrival at $\left[n x_{0}\right]$. These steps may be partitioned into $k$ disjoint sets, where the $i$ th set consists of those steps which occur before the first arrival at $\left[n\left(x_{0}+(k-i) / n\right)\right]$, but after the first arrival at $\left[n\left(x_{0}+(k-i+1) / n\right)\right]$, and these sets obviously have an independent numbers of elements. The generating function of the number of elements in the $i$ th set is evidently $G_{i}(s)$ since the situation is the same as in the proof of Theorem 1.2. The generating function of the total number of steps is therefore $\prod_{i=1}^{k} G_{i}(s)$. We again introduce Laplace transforms by setting $k=n x$ and $s=e^{-\lambda / n}$. The result may be written

$$
L(x, n, \lambda)=\prod_{i=1}^{n x}\left(1-\frac{1-e^{-\lambda / n}}{i\left(1-e^{-\lambda / n}\right)+1}\right) .
$$

The limit as $n$ becomes large is easily found, for one has, even without the assumption that $x=k / n$,

$\lim L(x, n, \lambda)$

$$
\begin{aligned}
& =\lim _{n \rightarrow \infty} \exp \sum_{i=1}^{[n x]}-\frac{\frac{\lambda}{n}+O\left(\frac{\lambda}{n}\right)^{2}}{1+i\left(\frac{\lambda}{n}-O\left(\frac{\lambda}{n}\right)^{2}\right)}+[n x] O\left(1-e^{-\lambda / n}\right)^{2} \\
& =\lim _{n \rightarrow \infty} \exp \sum_{i=1}^{[n x]}-\frac{1}{n}\left(\frac{\lambda}{1+i\left(\frac{\lambda}{n}\right)}\right)=\exp \int_{0}^{x}-\frac{\lambda}{1+\lambda y} d y=(\lambda x+1)^{-1},
\end{aligned}
$$

and the convergence is uniform in finite intervals of $\lambda$ and $x$. Under the assumption that we are dealing with a Markov process, the generator of this part of the process is thus $\left.\left.(d / d x)(\lambda x+1)^{-1}\right]_{x=0}=-\lambda=(d / d y) e^{-\lambda y}\right]_{y=0}$, and for the whole process the generator is to be found by differentiating $(\lambda x+1)^{-1} \exp (-\lambda y /(1+\lambda x))$ since the two components are independent. In this way one derives

$$
\left.\frac{d}{d x}(\lambda x+1) \exp _{1} \frac{-\lambda y}{1+\lambda x}\right]_{x=0}=\left(-\lambda+\lambda^{2} y\right) e^{-\lambda y}=\left(y \frac{d^{2}}{d y^{2}}+\frac{d}{d y}\right) e^{-\lambda y}
$$

indicating that generator has the form $y d^{2} / d y^{2}+d / d y$. For this generator, 0 is an entrance boundary and no boundary conditions may be imposed. It is then easy to see, as above, that the process must indeed be the diffusion with this generator.

It remains to establish, however, that the higher dimensional limiting joint distributions exist and are those of a Markov process. Let $X(x)$ denote a version of the limit process (if any) for $0 \leqq x \leqq-x_{0}$, and let $T_{x} f(y)$ denote 
$E\left(f\left(X\left(x_{1}+x\right)\right) \mid X\left(x_{1}\right)=y\right), 0<x_{1}<x_{1}+x<-x_{0}$. Then it has been shown that $T_{x}\left(e^{-\lambda y}\right)=\left(-\lambda+\lambda^{2} y\right) e^{-\lambda y}$, and it follows by uniform limits that the distributions converge weakly (weak $*$ ) at each $x$. We consider now approximating Riemann sums, with subdivisions of length $\Delta$, for the joint distributions in a product of intervals $\left[\beta_{1, i}, \beta_{2, i}\right), \beta_{1, i}>0,1 \leqq i<k$, at parameter values $0<x_{1}<\cdots<x_{k}<-x_{0}$. When $n$ is large, it follows as in the proof of Theorem 2.1 that the conditional probabilities

$P\left(\frac{1}{n} S\left(\left[n x_{0}\right],\left[n \alpha_{0}\right],\left[n\left(x_{0}+x_{1}+x\right)\right]\right) \in[y, y+\Delta) \mid \frac{1}{n} S\left(\left[n x_{0}\right],\left[n \alpha_{0}\right],\left[n\left(x_{0}+x_{1}\right)\right]\right)=\alpha\right)$

converge uniformly in $(\alpha, x)$ for $\alpha$ and $x$ in intervals of length $\Delta$ bounded away from 0 . In fact, the additional component of the process leading to the term $+d / d y$ in the generator does not involve the initial value $\alpha$, and since the limit distributions are known to be continuous in $(0, \infty)$ the asserted uniformity follows from that of the convergence in Theorem 2.1 and of $L(x, n, \lambda)$. It is therefore clear that the approximating sums are uniformly near to the actual joint distributions for all large $n$ (and small $\Delta$ ), and that their limits are the corresponding Riemann sums for the joint distributions of the required diffusion process. It is then seen by choosing $\Delta$ small that the joint distributions of the random walk sojourn processes $(1 / n) S\left(\left[n x_{0}\right],\left[n \alpha_{0}\right],\left[n\left(x_{0}+x\right)\right]\right)$ converge to those of the required diffusion process for $0<x<-x_{0}$. Since the same argument shows the Markovian nature of the limit joint distributions about $x=-x_{0}$ the proof is complete.

2. The effect of the last section was to establish a weak convergence of the joint distributions of certain discrete Markov chains to the joint distributions of corresponding diffusion processes. It is well known that with a suitable change of parameter the joint distributions of the random walks from which these Markov chains are defined converge to those of Brownian motion, and it is therefore to be expected that the limit diffusion processes have a direct interpretation in terms of Brownian motion. To establish this interpretation, we strengthen the weak convergence of distributions to an a.s. convergence based on the construction of [10]. This necessitates two adjustments-first, the construction in [10] was possible only for random walks of step size $2^{-k}, k>0$, and second, it is necessary to change the scale of the step duration (i.e., the time parameter) as well as the size, and the durations leading to Brownian motion from steps of size $2^{-k}$ are well known to be $2^{-2 k}$. We review quickly some notation and basic material from [10]. Let $R_{k}\left(n \alpha_{k}\right)$, for each $k \geqq 1$, be the symmetric random walk with step size $\beta_{k}=2^{-k}$ and duration $\alpha_{k}=2^{-2 k}$. Let $M_{k}, k \geqq 2$, be the mapping of the sample paths of $R_{k}$ onto those of $R_{k-1}$ determined by removing from each $R_{k}$-path all steps with values not of the form $m \beta_{k-1}$, then removing all the remaining steps which are equal in value to their immediate predecessors, and 
finally reparametrizing the remaining sequence of steps by assignment of duration $\alpha_{k-1}$ to each (3). The conclusion of [10] is that the $R_{k}$ and $M_{k}$ make up a projective limit probability space $\left(\Omega_{\infty}, F_{\infty}, P_{\infty}\right)$ on which the $R_{k}$-paths (taken either as step functions or as polygonal lines) converge a.s. uniformly in all finite time intervals to the paths of a Brownian motion $R_{\infty}(t), t \geqq 0$, defined on $\left(\Omega_{\infty}, F_{\infty}, P_{\infty}\right)$. It is sometimes conceptually easier to view this construction in reverse, by starting with a Brownian motion process. The random walk $R_{k}$ is then defined by reparametrizing the sequence of arrivals of the process on the lattice $m \beta_{k}$, excluding "successive" repetitions. More explicitly, let the given Brownian motion process be denoted by $R_{\infty}(t)$, and define inductively a sequence of stopping times $T(n, k)$ by

$$
T(1, k)=\inf _{t}\left|R_{\infty}(t)\right| \geqq \beta_{k} \text {, and } T(n+1, k) \quad=\inf _{t>T(n, k)}:\left|R_{\infty}(t)-R_{\infty}(T(n, k))\right| \geqq \beta_{k} .
$$

Then, except on a set of probability 0 , where some $T(n, k)$ fails to be finite, we define $R_{k}\left(n \alpha_{k}\right)=R_{\infty}(T(n, k))$ for all $k$ and $n$. Regarded in these terms the next lemma is almost obvious.

Definition 2.1. Let $S\left(k_{1}, k_{2}, b, m, r\right)$, for integer-valued arguments such that $b \leqq 0,0<k_{1} \leqq k_{2}, 0<m$, and $(b+1) \beta_{k_{1}} \leqq r \beta_{k_{2}}$, be the number of steps of $R_{k_{2}}$ from $r \beta_{k_{2}}$ to $(r+1) \beta_{k_{2}}$ by the end of the $(m+1)$ th step of $R_{k_{1}}$ from $b \beta_{k_{1}}$ to $(b+1) \beta_{k_{1}}$, as defined on the joint probability space $\left(\Omega_{\infty}, F_{\infty}, P_{\infty}\right)$, and let $S\left(k_{1}, k_{2}, b, 0, r\right)$ for $b \beta_{k_{1}} \leqq r \beta_{k_{2}}$ be the number of steps of $R_{k_{2}}$ from $r \beta_{k_{2}}$ to $(r+1) \beta_{k_{2}}$ before the first arrival of $R_{k_{1}}$ at $b \beta_{k_{1}}$.

LeMmA 2.1. For fixed $k_{1}, b, m>0$, and $x=n \beta_{K}$ where $n \beta_{K} \geqq(b+1) \beta_{k_{1}}$, the random variables

$$
V\left(k_{2}, x\right)=\beta_{k_{2}}\left(S\left(k_{1}, k_{2}, b, m, x \beta_{k_{2}}^{-1}\right)+S\left(k_{1}, k_{2}, b, m, x \beta_{k_{2}}^{-1}-1\right)+\delta\right),
$$

where $\delta=0$ if $x>0$ or 1 if $x \leqq 0$, form a martingale in $k_{2} \geqq \max \left(K, k_{1}\right)$. For $m=0$ the statement holds with $x \geqq b \beta_{k_{1}}$.

Proof. Let us first rephrase the lemma. For $m>0$ and $x>0$, clearly $\beta_{k_{2}}^{-1} V\left(k_{2}, x\right)$ is the total number of arrivals of $R_{k_{2}}$ at $x$ before the $(m+1)$ th step of $R_{k_{1}}$ from $b \beta_{k_{1}}$ to $(b+1) \beta_{k_{1}}$, while for $(b+1) \beta_{k_{1}} \leqq x \leqq 0$ this number of arrivals is equal to $\beta_{k_{2}}^{-1} V\left(k_{2}, x\right)+1$. Hence the lemma states that the total numbers of arrivals by $R_{k_{2}}$ at $x$ before the $(m+1)$ th step of $R_{k_{1}}$ from $b \beta_{k_{1}}$ to $(b+1) \beta_{k_{1}}$, when multiplied by $\beta_{k_{2}}$, form a martingale as soon as $x$ is accessible for $R_{k_{2}}$. A similar interpretation is evident for $m=0$.

To prove the assertion, let there be given for some $k_{2} \geqq \max \left(K, k_{1}\right)$ the quantities $R_{k_{2}}\left(n \alpha_{k_{2}}\right)$ for all $n$ between 0 and the integer ar which $R_{k_{1}}$ completes its $(m+1)$ th step from $b \beta_{k_{1}}$ to $(b+1) \beta_{k_{1}}(m>0)$, (resp. first arrives at

(3) We assume that for each $R_{k}$ the set of probability 0 mentioned at the start of $\S 1$ has been removed from the space. It should be noted that $\alpha_{k}$ has no connection with the notation $\alpha_{0}$ which is used to denote an initial value of a process. 
$b \beta_{k_{1}},(m=0)$ ), where $R_{k_{1}}$ is derived from $R_{k_{2}}$ by the mapping $M_{k_{2}} M_{k_{2}-1} \cdots M_{k_{1}+1}$. The number of arrivals of $R_{k_{2}+1}$ at $x$ is then a sum of $\beta_{k_{2}}^{-1} V\left(k_{2}, x\right),(x>0)$, or $\beta_{k_{2}}^{-} V\left(k_{2}, x\right)+1,(x \leqq 0)$, conditionally independent and identically distributed random variables equalling the numbers of arrivals of $R_{k_{2}+1}$ at $x$ starting with each arrival of $R_{k_{2}}$ at $x$ and before the next step of $R_{k_{2}}$. These variables have the distribution of $1+V$, where $V$ is geometrically distributed with generating function $(2-s)^{-1}$. Their expectation is accordingly 2 , and since $\beta_{k_{2}}^{-1} \beta_{k_{2}+1}=2^{-1}$ the lemma follows upon taking the expectation over all (given) sets of values of $R_{k_{2}}\left(n \alpha_{k_{2}}\right)$ compatible with the given value of $V\left(k_{2}, x\right)$.

We are now able to prove an a.s. convergence sufficient for the present purposes.

LEMMA 2.2. For $b \leqq 0 \leqq m, 0<k_{1}$, and all $x$ of the form $n \beta_{K} \geqq(b+1) \beta_{k_{1}}$ (if $m>0)$, or $\geqq b \beta_{k_{1}}$ (if $m=0$ ), the limits $\lim _{k_{2} \rightarrow \infty} \beta_{k_{2}} S\left(k_{1}, k_{2}, b, m, x \beta_{k_{2}}^{-1}\right)$ exist simultaneously a.s.

Proof. Since the random variables $V\left(k_{2}, x\right)$ for $b, m$, and $k_{1}$ fixed, are nonnegative and have bounded expectations, as is easily seen, it follows from Lemma 2.1 and a martingale convergence theorem that the $\operatorname{limits}_{\lim _{k_{2} \rightarrow \infty}} V\left(k_{2}, x\right)$ exist a.s. If for a certain $k_{2}$ both $V\left(k_{2}, x\right)$ and $R_{k_{2}}\left(n \alpha_{k_{2}}\right)$, for the range of $n$ considered in the proof of Lemma 2.1, are given, then $S\left(k_{1}, k_{2}+1, b, m, x \beta_{k_{2}+1}^{-1}\right)$ is the sum of $\beta_{k_{2}}^{-1} V\left(k_{2}, x\right),(x>0)$, or $\beta_{k_{2}}^{-1} V\left(k_{2}, x\right)+1$, $(x \leqq 0)$, conditionally independent and identically distributed random variables equalling the numbers of steps of $R_{k_{2}+1}$ from $x$ to $x+\beta_{k_{2}+1}$ between the successive arrivals of $R_{k_{2}}$ at $x$. These have the distribution of a sum of $1+V$ independent Bernouilli random variables equal to 0 or 1 with $p=\frac{1}{2}$, where $V$ is independent of these and has the generating function $(2-s)^{-1}$. The generating function of this composite random variable is thus $(1+s) /(3-s)$, and its mean and variance are 1 and $\frac{1}{2}$. The conditional mean and variance of $\beta_{k_{2}+1} S\left(k_{1}, k_{2}+1, b, m, x \beta_{k_{2}+1}^{-1}\right)$ are therefore $\frac{1}{2} V\left(k_{2}, x\right)$ and $\frac{1}{8} \beta_{k_{2}} V\left(k_{2}, x\right)$, if $x>0$, or $\frac{1}{2} V\left(k_{2}, x\right)+\beta_{k_{2}+1}$ and $\frac{1}{8} \beta_{k_{2}} V\left(k_{2}, x\right)+\frac{1}{2} \beta_{k_{2}+1}^{2}$, if $x \leqq 0$. Since $\sum \beta_{k_{2}}=1$, it follows from the Borel-Cantelli lemma (slightly generalized) that a.s.

$$
\lim _{k_{2} \rightarrow \infty} \beta_{k_{2}} S\left(k_{1}, k_{2}, b, m, x \beta_{k_{2}}^{-1}\right)=\frac{1}{2} \lim _{k_{2} \rightarrow \infty} V\left(k_{2}, x\right) .
$$

The convergence is extended to all $b, m, k_{1}$, and $x$ by noting that only countably many possibilities are involved.

The effect of Lemma 2.2 is to define for each $k_{1}, b$, and $m$, a limit process on the diadic rationals $x \geqq(b+1) \beta_{k}$ (resp. $x \geqq b \beta_{k_{1}}$ ). By using the arguments of $\S 1$ it will be shown that this process is the restriction to a diadic rational parameter of a diffusion process of the type considered there, although with a nondegenerate initial distribution in the case of $m>0$.

LEMMA 2.3. The process 


$$
s\left(k_{1}, b, m ; x\right)=\lim _{k_{2} \rightarrow \infty} \beta_{k_{2}}\left(S\left(k_{1}, k_{2}, b, m, \beta_{k_{2}}^{-1}\left((b+1) \beta_{k_{1}}+x\right)\right)\right)
$$

for $b<0, m>0$, and diadic rational $x \geqq 0$ (where the definition is to be adjusted by using 0 if the limit for any $x$ does not exist) is the restriction of a diffusion process as defined in Corollary 1.3 with $x_{0}=(b+1) \beta_{k_{1}}$ and an initial distribution of $\alpha_{0}$ equal to that of $s\left(k_{1}, b, m ; 0\right)$. For $b=0$ the same statement holds with $x_{0}=0$. Similarly,

$$
s\left(k_{1}, b, 0 ; x\right)=\lim _{k_{2} \rightarrow \infty} \beta_{k_{2}}\left(S\left(k_{1}, k_{2}, b, 0, \beta_{k_{2}}^{-1}\left(b \beta_{k_{1}}+x\right)\right)\right)
$$

exists for diadic rational $x \geqq 0$ and is a realization of the process of Corollary 1.3 with $x_{0}=b \beta_{k_{1}}$ and $\alpha_{0}=0$.

Proof. The lemma follows directly from the proofs of $\S 1$. Suppose, in fact, that in Corollary 1.1 " $R(n)$ ", is replaced by $\beta_{k_{2}}^{-1} R_{k_{2}}\left(n \alpha_{k_{2}}\right)$, " $b$ "' by $(b+1) \beta_{k_{1}} \beta_{k_{2}}^{-1}$, and ' $m$ "' by $S\left(k_{1}, k_{2}, b, m,(b+1) \beta_{k_{1}} \beta_{k_{2}}^{-1}\right)$, (for $m>0$ ), which we denote temporarily by $m^{*}$. It is clear that, in the notation of the proof of Theorem $1.1, m^{*}$ is measurable over $F(i)$ for each $i$ (we allow here any $k$ such that $(b+1) \beta_{k_{1}} \beta_{k_{2}}^{-1} \leqq k$ ). This is evidently the only fact required of an initial value in order to establish, by the same reasoning as given there, the Markovian character of $S\left((b+1) \beta_{k_{1}} \beta_{k_{2}}^{-1}, m^{*}, k\right), k \geqq 0$, and the form of the transition function. The passage to the limit in $k_{2}$ now proceeds just as in Theorem 1.2 and corollaries, except that we have to replace $n$ by $\beta_{k_{2}}^{-1},\left[n x_{0}\right]$ by $(b+1) \beta_{k_{1}} \beta_{k_{2}}^{-1}$, and $\left[n \alpha_{0}\right]$ by $m^{*}$, and then use Lemma 2.2 to the effect that tie distribution of the initial value $\beta_{k_{2}} m^{*}$ converges to that of $s\left(k_{1}, b, m ; 0\right)$. The case of $s\left(k_{1}, b, 0 ; x\right)$ is analogous, except that the index of the first arrival at $b \beta_{k_{1}}$ is measurable over $F(i)$ for any $k$ such that $b \beta_{k_{1}} \beta_{k_{2}}^{-1} \leqq k$, whence the initial value may be taken at $x_{0}=b \beta_{k_{1}}$, and is accordingly 0 .

DEFINITION 2.2. Let $s\left(k_{1}, b, m ; x\right), x \geqq 0$, be defined as the extension by continuity of the process of Lemma 2.3 to all $x \geqq 0$ whenever the former is uniformly continuous in all finite intervals of diadic rational $x$, and set $s\left(k_{1}, b, m ; x\right)=0$ otherwise.

The conclusion of Lemma 2.3 is that $s\left(k_{1}, b, m ; x\right), m>0$, is a diffusion process with generator $y d^{2} / d y^{2}+d / d y$ for $0 \leqq x<-(b+1) \beta_{k_{1}}$, and $y d^{2} / d y^{2}$ for $-(b+1) \beta_{k} \leqq x$, where 0 is an absorbing barrier. This process has a nondegenerate initial distribution, and to derive the process with initial value $\alpha_{0}>0$ fixed it is necessary to take a further limit in $k_{1}$. Before doing this, however, we show that the present process is already a sojourn density process of the Brownian motion $R_{\infty}(t)$

Definition 2.3. For $b \leqq 0<m$, let $T\left(k_{1}, b, m\right)$ denote inf : $_{t}$ in $0<\tau<t$ there are at least $m+1$ disjoint passages of $R_{\infty}(\tau)$ from $b \beta_{k_{1}}$ to $(b+1) \beta_{k_{1}}$, where a "passage"' is an interval $\left(\tau_{1}, \tau_{2}\right)$ such that $R_{\infty}\left(\tau_{1}\right)=b \beta_{k_{1}}, R_{\infty}\left(\tau_{2}\right)=(b+1) \beta_{k_{1}}$. Equi- 
valently, $T\left(k_{1}, b, m\right)=\inf _{t}$ : in $0 \leqq \tau \leqq t$ there are at least $m+1$ steps of $R_{k_{1}}\left(n \alpha_{k_{1}}\right)$ from $b \beta_{k_{1}}$ to $(b+1) \beta_{k_{1}}$ where $\tau$ is the time parameter of $R_{\infty}$ in $\left(\Omega_{\infty}, F_{\infty}, P_{\infty}\right)$ and maps onto the parameter of $R_{k_{1}}$ as in [10]. Lastly, let $T\left(k_{1}, b, 0\right)$ denote the first passage time of $R_{\infty}$ to $b \beta_{k_{1}}$.

THEOREM 2.1. Let $I_{(a, b)}(x)=0$ or 1 according to whether $x \notin(a, b)$ or $x \in(a, b)$. Then for $0<m$,

$$
s\left(k_{1}, b, m ; x\right)=\frac{1}{2} \frac{d}{d x} \int_{0}^{T\left(k_{1}, b, m\right)} I_{\left(-\infty,(b+1) \beta_{k_{1}}+x\right)}\left(R_{\infty}(t)\right) d t
$$

for all $x \geqq 0$ a.s., while for $0=m$, a.s. for $x \geqq 0$,

$$
s\left(k_{1}, b, 0 ; x\right)=\frac{1}{2} \frac{d}{d x} \int_{0}^{T\left(k_{1}, b, 0\right)} I_{\left(-\infty, b \beta_{k_{1}}+x\right)}\left(R_{\infty}(t)\right) d t .
$$

In other words, $2 s\left(k_{1}, b, m ; x\right)$ is a sojourn density process of $R_{\infty}(t)$.

Proof. For the course of this proof we set $(b+1) \beta_{k_{1}}=\gamma$. Let there be introduced the "approximate $\frac{1}{2}$ sojourn density processes" based on the random walks $R_{k_{2}}$ and defined as follows:

$$
s\left(k_{1}, k_{2}, b, m ; x\right)=\beta_{k_{2}} S\left(k_{1}, k_{2}, b, m, r\right) \text { for } \gamma \leqq r \beta_{k_{2}} \leqq x+\gamma<(r+1) \beta_{k_{2}}
$$

if $m>0$, or similarly with $b \beta_{k_{1}}$ in place of $\gamma$ if $m=0$. It follows from known results $[11$, p. 240$]$ that $\int_{0}^{T\left(k_{1}, b, m\right)} I_{(-\infty, y)}\left(R_{\infty}(t)\right) d t$ is continuous in $y$ a.s. at each $y$. It is therefore clear from the uniform pathwise convergence of [10] that, in the case $m>0$, for each $y>\gamma$ one has

$$
\lim _{k_{2} \rightarrow \infty} 2 \int_{0}^{y-\gamma} s\left(k_{1}, k_{2}, b, m ; x\right) d x=\int_{0}^{T\left(k_{1}, b, m\right)} I_{(\gamma, y)}\left(R_{\infty}(t)\right) d t
$$

a.s., where the factor 2 appears because only the steps in the positive direction are counted from $R_{k_{2}}$, and the number of these is a.s. asymptotically $\frac{1}{2}$ of the total number of steps of $R_{k_{2}}$ which start in $(\gamma, y)$ during $\left(0, T\left(k_{1}, b, m\right)\right)$. Similarly, for $m=0$, a.s. for each $y$ :

$$
\lim _{k_{2} \rightarrow \infty} 2 \int_{0}^{y-b \beta_{k_{1}}} s\left(k_{1}, k_{2}, b, 0 ; x\right) d x=\int_{0}^{T\left(k_{1}, b, 0\right)} I_{\left(b \beta_{k_{1}}, y\right)}\left(R_{\infty}(t)\right) d t .
$$

If it be shown that for each diadic rational $y \geqq \gamma$, for the case $m>0$, the left side of (2.1) is equal to $2 \int_{0}^{y-\gamma} s\left(k_{1}, b, m ; x\right) d x$ a.s., then it will follow from the monotonicity in $y$ of $\int_{0}^{T\left(k_{1}, b, m\right)} I_{(\gamma, y)}\left(R_{\infty}(t)\right) d t$ that a.s. for all $y \geqq \gamma$

$$
2 \int_{0}^{y-\gamma} s\left(k_{1}, b, m ; x\right) d x=\int_{0}^{T\left(k_{1}, b, m\right)} I_{(\gamma, y)}\left(R_{\infty}(t)\right) d t,
$$

and the theorem in this case is then obtained by differentiation. In the case $m=0$, the same method succeeds with $\gamma$ replaced by $b \beta_{k_{1}}$. Accordingly, let it be supposed 
that for a certain $y$ this fails with positive probability. Then there exists an $\varepsilon>0$ such that for all $k_{2}$ sufficiently large

$$
P\left\{\int_{0}^{y-\gamma}\left|s\left(k_{1}, k_{2}, b, m ; x\right)-s\left(k_{1}, b, m ; x\right)\right| d x>\varepsilon\right\}>\varepsilon .
$$

It follows, however, from the convergence of the joint distributions of $s\left(k_{1}, k_{2}, b, m ; x\right)$ to those of the diffusion $s\left(k_{1}, b, m ; x\right)$, that the joint distributions for products of intervals $\left[\beta_{1, i}, \beta_{2, i}\right), 1<i \leqq k$, and for $x_{i}$ on the lattice $\left\{x_{n}=n \delta_{1}\right\}$ converge to those of the diffusion for each $\delta_{1}$, and since the diffusion is continuous, one sees, by the standard argument based on the fact that the variance of the transition function is uniformly small for small values of the transition parameter, that a large increment of the process $s\left(k_{1}, k_{2}, b, m ; x\right)$ in a range $\left(x_{1}, x_{2}\right)$ with $\left|x_{2}-x_{1}\right|<\delta_{1}$, would "imply" a corresponding large increment on two successive points $x_{n}=n \delta_{1}$, and hence finally that for $\delta_{2}>0$ given, $\delta_{1}>0$ sufficiently small, and $k_{2}$ sufficiently large, with $0 \leqq x_{1}<x_{2}<y-\gamma$,

$$
P\left\{\sup _{\left|x_{1}-x_{2}\right|<\delta_{1}}\left|s\left(k_{1}, k_{2}, b, m ; x_{1}\right)-s\left(k_{1}, k_{2}, b, m ; x_{2}\right)\right|>\delta_{2}\right\}<\delta_{2} .
$$

The integral inequality above leads to a contradiction with this fact. It implies that with probability $\varepsilon$,

$$
\sup _{x}\left|s\left(k_{1}, k_{2}, b, m ; x\right)-s\left(k_{1}, b, m ; x\right)\right|>\frac{\varepsilon}{y-\gamma},
$$

and hence, setting $\delta_{2}=\min (\varepsilon / 2, \varepsilon / 2(y-\gamma))$, we would have for all $k_{2}$ sufficiently large a probability of at least $\varepsilon / 2$ that there exist an interval $I$ of length $\delta_{1}$ in $(0, y-\gamma)$ for which

$$
\left|s\left(k_{1}, k_{2}, b, m ; x\right)-s\left(k_{1}, b, m ; x\right)\right|>\frac{\varepsilon}{2(y-\gamma)}
$$

holds for all $x$ in $I$. Such an infinite collection of intervals must contain some diadic rational infinitely often, and this contradicts the convergence of $s\left(k_{1}, k_{2}, b, m ; x\right)$ to $s\left(k_{1}, b, m ; x\right)$ at all diadic rationals a.s. The proof is thus complete for the case $m>0$, and the case $m=0$ is treated analogously.

The next theorem is the existence theorem for the sojourn density processes of $R_{\infty}(t)$ corresponding to the limit distributions of Theorem 1.2 and corollaries. At first, the complete meaning of the random time intervals involved in defining these processes must be left partially open since it turns out that they are of the form

$$
\left(0, \underset{t}{\inf :} \frac{1}{2} \frac{d}{d x} \int_{0}^{t} I_{\left(-\infty, x_{0}\right)}\left(R_{\infty}(\tau)\right) d \tau>\alpha_{0}\right)
$$

and the existence of this derivative for all $t$ has not been established here. However, if one refers to [12] or [14] for the existence of the derivative, it seems fairly clear from the start that the above is the correct interpretation. 
THEOREM 2.2(4). For $\alpha_{0} \geqq 0$ and $x_{0} \leqq 0$, let $T\left(x_{0}, \alpha_{0}\right)$ denote

$$
\inf _{t}: \frac{d}{d x} \int_{0}^{t} I_{(-\infty, x)}\left(R_{\infty}(\tau)\right) d \tau>\alpha_{0} .
$$

Then $\frac{1}{2} f\left(x_{0}+x, T\left(x_{0}, \alpha_{0}\right), w\right)$ is the diffusion process of Corollary 1.3.

More elaborately, the following statements hold. The limit

$$
\lim _{k \rightarrow \infty} s\left(k,\left[x_{0} \beta_{k}^{-1}\right]-1,\left[\alpha_{0} \alpha_{k}^{-1}\right] ; x\right)
$$

exists a. s. uniformly in $x \geqq 0$. Let $s\left(x_{0}, \alpha_{0} ; x\right)$ denote this limit, or 0 when it fails for some $x$ to exist. Then $s\left(x_{0}, \alpha_{0} ; x\right)$ is a realization of the process of Corollary 1.3. Moreover, it is a sojourn density process of $R_{\infty}(t)$, in the sense that the limit $T\left(x_{0}, \alpha_{0}\right)=\lim _{k \rightarrow \infty} T\left(k,\left[x_{0} \beta_{k}^{-1}\right]-1,\left[\alpha_{0} \beta_{k}^{-1}\right]\right)$ exists a.s. and, in terms of the notation $f(x, t, w)$ used in the introduction,

$$
\begin{aligned}
s\left(x_{0}, \alpha_{0} ; x\right) & =\frac{1}{2} \frac{d}{d x} \int_{0}^{T\left(x_{0}, \alpha_{0}\right)} I_{\left(-\infty, x_{0}+x\right)}\left(R_{\infty}(t)\right) d t & \\
& =\frac{1}{2} f\left(x_{0}+x, T\left(x_{0}, \alpha_{0}\right), w\right) & \text { for all } x \text { a. } s .
\end{aligned}
$$

Finally,

$$
T\left(x_{0}, \alpha_{0}\right)=\inf _{t}: \frac{1}{2} \frac{d}{d x} \int_{0}^{t} I_{\left(-\infty, x_{0}\right)}\left(R_{\infty}(\tau)\right) d \tau>\alpha_{0}
$$

a.s., where the infimum is over those $t$ for which the derivative exists.

Proof. The proof is unfortunately rather complicated. We shall suppose at first that $x_{0}$ and $\alpha_{0}$ are diadic rationals, which for large $k$ allows us to avoid the "square brackets" notation, and that $\alpha_{0}$ is strictly positive. The main obstacle in the proof is to show that the limit $T\left(x_{0}, \alpha_{0}\right)$ exists a.s. If this be granted, then evidently $R_{\infty}\left(T\left(x_{0}, \alpha_{0}\right)\right)=x_{0}$ holds a.s. and the contribution to the sojourn times for $t$ between $T\left(x_{0}, \alpha_{0}\right)$ and $T\left(k, x_{0} \beta_{k}^{-1}-1, \alpha_{0} \beta_{k}^{-1}\right)$ vanishes outside a neighborhood of $x_{0}$ tending to $\left\{x_{0}\right\}$ as $k$ increases. However, the simplest way to prove the existence of $T\left(x_{0}, \alpha_{0}\right)$ seems to be by showing first, in essence, that the sojourn densities at $x_{0}$ up to time $T\left(k, x_{0} \beta_{k}^{-1}-1, \alpha_{0} \beta_{k}^{-1}\right)$, which we shall abbreviate to $T(k)$, converge to $2 \alpha_{0}$ a.s. Recalling that $T(k)$ is the time of the $\alpha_{0} \beta_{k}^{-1}$ th disjoint passage of $R_{\infty}$ from $x_{0}-\beta_{k}$ to $x_{0}$, it is easy to see from the strong law of large numbers that there are a.s. asymptotically $2 \alpha_{0} \beta_{k}^{-1}$ passages of $R_{\infty}(t)$ from $x_{0}$ to $\left\{x_{0} \pm \beta_{k}\right\}$ up to time $T(k)$. We wish to show that $s\left(k, x_{0} \beta_{k}^{-1}-1, \alpha_{0} \beta_{k}^{-1} ; 0\right)$ converges to $\alpha_{0}$ a.s., and it is convenient to consider the total duration of the passages from

(4) Part of this theorem was presented to the Society August 30, 1962 under the title $A$ sojourn density process of Brownian motion. 
$x_{0}$ to $\left\{x_{0} \pm \beta_{k}\right\}$ (rather than only from $x_{0}$ to $x_{0}+\beta_{k}$, or from $x_{0}$ to $x_{0}+\beta_{k}$ and from $x_{0}=\beta_{k}$ to $x_{0}$, etc.). Let $D\left(k_{1}, k_{2}\right)$ denote the total duration of passages of $R_{\infty}(t)$ from $x_{0}$ to $\left\{x_{0} \pm \beta_{k_{2}}\right\}$ up to time $T\left(k_{1}\right)$. It will be shown that $\lim _{k_{2} \rightarrow \infty} \beta_{k_{2}}^{-1} D\left(k_{1}, k_{2}\right)=2 s\left(k_{1}, x_{0} \beta_{k_{1}}^{-1}, \alpha_{0} \beta_{k_{1}}^{-1} ; 0\right)$ a.s.; this last expression we shall abbreviate by $2 s\left(k_{1}\right)$, and that $\lim _{k_{1} \rightarrow \infty} \lim _{k_{2} \rightarrow \infty} \beta_{k_{2}}^{-1} D\left(k_{1}, k_{2}\right)=2 \alpha_{0}$ a.s. Combining these results, one reaches the desired conclusion immediately $\left({ }^{5}\right)$.

By Lemma 2.3, $\beta_{k_{2}}$ times the number of passages from $x_{0}$ to $x_{0}+\beta_{k_{2}}$ up to time $T\left(k_{1}\right)$ converges to $s\left(k_{1}\right)$ a.s. This clearly implies that $\beta_{k_{2}}$ times the corresponding number of passages from $x_{0}$ to $x_{0}-\beta_{k_{2}}$ up to time $T\left(k_{1}\right)$ converges a.s. to the same limit. When the number of passages from $x_{0}$ to $\left\{x_{0} \pm \beta_{k_{2}}\right\}$ up to time $T\left(k_{1}\right)$ is given, their durations are independent and identically distributed, and are, in fact, independent of the given information altogether. It follows from [10, p. 222 and Theorem 3] that each of these durations has mean $\alpha_{k_{2}}$ and variance less than $\frac{2}{3} \alpha_{k_{2}}^{2}$. Therefore, the conditional mean of $\beta_{k_{2}}^{-1} D\left(k_{1}, k_{2}\right)$ converges to $s\left(k_{1}\right)$ a.s., and the conditional variance of the same quantity is asymptotically not greater than $\frac{2}{3} \beta_{k_{2}}^{3} s\left(k_{1}\right)$. It follows easily from these facts that $\lim _{k_{2} \rightarrow \infty} \beta_{k_{2}}^{-1} D\left(k_{1}, k_{2}\right)=2 s\left(k_{1}\right)$ a.s.

The other statement is less immediate. Let $N\left(k_{1}, k_{2}\right)$ denote the number of (disjoint) passages from $x_{0}$ to $\left\{x_{0} \pm \beta_{k_{2}}\right\}$ by time $T\left(k_{1}\right)$, and set $M\left(k_{1}, k_{2}\right)=$ $\beta_{k_{2}} N\left(k_{1}, k_{2}\right)$. We suppose that $M\left(k_{1}, k_{1}\right)$ is given, and compute the conditional mean and variance of $M\left(k_{1}, k_{2}\right), k_{1}<k_{2}$. If $M\left(k_{1}, k_{2}\right)$ is also given, then $N\left(k_{1}, k_{2}+1\right)$ is the sum of $N\left(k_{1}, k_{2}\right)$ independent and identically distributed random variables equalling the numbers of passages from $x_{0}$ to $\left\{x_{0} \pm \beta_{k_{2}+1}\right\}$ during the $N\left(k_{1}, k_{2}\right)$ respective passages from $x_{0}$ to $\left\{x_{0} \pm \beta_{k_{2}}\right\}$. These numbers have the generating function $(2-s)^{-1}$ considered before, and in particular have mean and variance both equal to 2 . Therefore,

and

$$
E\left(M\left(k_{1}, k_{2}+1\right) \mid M\left(k_{1}, k_{2}\right)\right)=M\left(k_{1}, k_{2}\right)
$$

$$
E\left(M^{2}\left(k_{1}, k_{2}+1\right) \mid M\left(k_{1}, k_{2}\right)\right)-E^{2}\left(M\left(k_{1}, k_{2}+1\right) \mid M\left(k_{1}, k_{2}\right)\right)=\frac{1}{2} \beta_{k_{2}} M\left(k_{1}, k_{2}\right) .
$$

Taking expectations over $M\left(k_{1}, k_{2}\right)$ on both sides, we have $E\left(M^{2}\left(k_{1}, k_{2}+1\right)\right)-E\left(M^{2}\left(k_{1}, k_{2}\right)\right)=\frac{1}{2} \beta_{k_{2}} M\left(k_{1}, k_{1}\right)$, when the latter is given, and therefore, still given $M\left(k_{1}, k_{1}\right)$,

$$
\begin{aligned}
E\left(M^{2}\left(k_{1}, k_{2}\right)\right) & =\sum_{k=k_{1}}^{k_{2}-1}\left(E\left(M^{2}\left(k_{1}, k+1\right)\right)-E\left(M^{2}\left(k_{1}, k\right)\right)\right)+M^{2}\left(k_{1}, k_{1}\right) \\
& <\frac{1}{2} \beta_{k_{1}} M\left(k_{1}, k_{1}\right)+M^{2}\left(k_{1}, k_{1}\right),
\end{aligned}
$$

whence the conditional variance of $M\left(k_{1}, k_{2}\right)$, given $M\left(k_{1}, k_{1}\right)$, is less than

(5) Added in proof. The argument may be shortened by replacing $D\left(k_{1}, k_{2}\right)$ by $a_{k_{2}} N\left(k_{1}, k_{2}\right)$ throughout. 
$\frac{1}{2} \beta_{k_{1}} M\left(k_{1}, k_{1}\right)$. From this it is seen that given $M\left(k_{1}, k_{1}\right), N\left(k_{1}, k_{2}\right)$ has mean $\beta_{k_{2}}^{-1} M\left(k_{1}, k_{1}\right)$ and variance less than $\beta_{k_{2}}^{-2} \beta_{k_{1}} M\left(k_{1}, k_{1}\right)$. The duration of each of the passages contributing to $N\left(k_{1}, k_{2}\right)$ has mean $\alpha_{k_{2}}$ and variance less than $\frac{2}{3} \alpha_{k_{2}}^{2}$, independently of $M\left(k_{1}, k_{1}\right)$. In order to estimate $D\left(k_{1}, k_{2}\right)$ we use the fact that such a sum $\sum_{i=1}^{V} U_{i}$, where the $V$ and $U_{i}$ are jointly independent, and where $E(U)=m_{1}, E(V)=m_{2}, \sigma^{2}(U)=\sigma_{1}^{2}, \sigma^{2}(V)=\sigma_{2}^{2}$, has mean $m_{1} m_{2}$ and variance $m_{1}^{2} \sigma_{2}^{2}+m_{2} \sigma_{1}^{2}$, as is easily shown by differentiating the composite function of the generating function of $V$ and the Laplace transform of $U_{i}$. Setting $m_{1}=\alpha_{k_{2}}, m_{2}=\beta_{k_{2}}^{-1} M\left(k_{1}, k_{1}\right), \sigma_{1}^{2}=\frac{2}{3} \alpha_{k_{2}}^{2}$, and $\sigma_{2}^{2}=\beta_{k_{2}}^{-2} \beta_{k_{1}} M\left(k_{1}, k_{1}\right)$, we find that $E\left(D\left(k_{1}, k_{2}\right) \mid M\left(k_{1}, k_{1}\right)\right)=\beta_{k_{2}} M\left(k_{1}, k_{1}\right)$, and that the conditional variance is less than $\left(\alpha_{k_{2}} \beta_{k_{1}}+\frac{2}{3} \beta_{k_{2}}^{3}\right) M\left(k_{1}, k_{1}\right)$. Therefore, $\beta_{k_{2}}^{-1} D\left(k_{1}, k_{2}\right)$ has conditional mean $M\left(k_{1}, k_{1}\right)$ and variance less than $\left(\beta_{k_{1}}+\frac{2}{3} \beta_{k_{2}}\right) M\left(k_{1}, k_{1}\right)$, and hence $\lim _{k_{2} \rightarrow \infty} \beta_{k_{2}}^{-1} D\left(k_{1}, k_{2}\right)$ has the same conditional mean and variance no greater than $\beta_{k_{1}} M\left(k_{1}, k_{1}\right)$. Since $\lim _{k_{1} \rightarrow \infty} M\left(k_{1}, k_{1}\right)=2 \alpha_{0}$ a.s., the Borel-Cantelli lemma implies as in Lemma 2.2 that $\lim _{k_{1} \rightarrow \infty} \lim _{k_{2} \rightarrow \infty} \beta_{k_{2}}^{-1} D\left(k_{1}, k_{2}\right)=2 \alpha_{0}$ a.s., as was to be shown.

It can now be proved that $\lim _{k \rightarrow \infty} T(k)=T\left(x_{0}, \alpha_{0}\right)$ exists a.s. and satisfies the assertion of the theorem. One notes first that $\lim _{0 u p_{k}} T(k)$ is finite a.s., for in the contrary case the total sojourn time up to time $T(k)$ would be unbounded in $k$, which would lead to a contradiction with $\lim _{k \rightarrow \infty} s(k)=\alpha_{0}$ a.s., in view of Lemma 2.3 and Theorem 2.1. Since $s(k)$ is a monotone function of $T(k)$ as $k$ varies, it follows that whenever $\liminf T(k)<T\left(k_{1}\right)<T\left(k_{2}\right)<\lim \sup T(k)$ then $s\left(k_{1}\right)=s\left(k_{2}\right)$. We now choose an $\varepsilon>0$ and distinguish two cases. If for a given path $w$ there is a sequence $k_{i}$ with $T\left(k_{i}\right) \downarrow \liminf T(k)$, while $\liminf T(k)+\varepsilon<\lim \sup T(k)$, then for $k_{i}$ large there is an increase of 0 in the sojourn density at $x_{0}$ during the interval $\left(T\left(k_{i}\right), \lim \sup T(k)\right)$, which has length at least $\varepsilon$. If, however, $\liminf T(k)+\varepsilon<\lim \sup T(k)$ but there is no such sequence $k_{i}$, then the interval $(\liminf T(k), \lim \sup T(k))$ evidently has the same property. Both $T(k)$, for any $k$, and $\liminf T(k)$ are stopping times for $R_{\infty}(t)$ and $R_{\infty}(\liminf (T(k)))=R_{\infty}(T(k))=x_{0}$ a.s. for all large $k$. It will be shown by using the strong Markov property of $R_{\infty}(t)$ that both of the above cases have probability 0 . It is enough to show that the probability of an increase of 0 in the sojourn density of a Brownian motion $R_{\infty}(t)$ at 0 during an interval $(0, \varepsilon), \varepsilon>0$, is 0 . This is shown in [12], but it is also a consequence of the fact that the number of returns to 0 of $R_{k}\left(n \alpha_{k}\right), 0 \leqq n \leqq \varepsilon \alpha_{k}^{-1}$, when multiplied by $(2 \varepsilon)^{-1 / 2} \beta_{k}$, has a distribution which converges to a truncated normal limit as $k$ becomes large [7, p. 83]. From this, it is clear that the duration of the passages of $R_{\infty}(t)$ from 0 to $\left\{ \pm \beta_{k}\right\}$ up to time $\varepsilon+\lim _{r \rightarrow \infty} L_{k, r}(\varepsilon)$ when multiplied by $(2 \varepsilon)^{-1 / 2} \beta_{k}$ has this same limit distribution, where $L_{k, r}$ is the "time lag" of [10, p. 221], and since $\lim _{k \rightarrow \infty} \lim _{r \rightarrow \infty} L_{k, r}(\varepsilon)=0$ a.s., [10], one sees that the increase of the density at 0 cannot be 0 with positive probability. Letting $\varepsilon$ decrease to 0 one has $\liminf T(k)$ $=\lim \sup T(k)$ a.s. and the existence of $T\left(x_{0}, \alpha_{0}\right)$ is proved. 
As noted at the start of the proof, the sojourn densities before time $T\left(x_{0}, \alpha_{0}\right)$ are thus identical with those before time $T(k)$ for $x$ outside a neighborhood of $x_{0}$ which tends to $\left\{x_{0}\right\}$ as $k$ increases a.s., and since the latter exist by Theorem 2.1 the former do likewise, and we have, therefore, proved the existence of $s\left(x_{0}, \alpha_{0} ; x\right), x>0$, as stated, except for the uniformity of the convergence near $x=x_{0}$ and the representation of $T\left(x_{0}, \alpha_{0}\right)$ as

$$
\inf _{t} \frac{1}{2} \frac{d}{d x} \int_{0}^{t} I_{\left(-\infty, x_{0}\right)}\left(R_{\infty}(\tau)\right) d \tau>\alpha_{0},
$$

but under the restriction that $x_{0}$ and $\alpha_{0}$ are diadic rationals with $\alpha_{0}>0$. From the existence of the sojourn density $2 s\left(x_{0}, \alpha_{0} ; x\right)$ for $x>0$ follow its existence at $x=0$ as a right derivative and also the fact that this derivative equals $2 \alpha_{0}$ a.s. But by applying the same result to the process for $x<0$, by using the symmetry about $x_{0}$ following the first arrival there, one sees that the left derivative has the same value a.s., and hence $2 s\left(x_{0}, \alpha_{0} ; x\right)$ exists as a sojourn density up to time $T\left(x_{0}, \alpha_{0}\right)$ for all $x$ a.s., and is a combination of two diffusion processes with oppositely directed parameters $x \geqq 0$ and $x \leqq 0$, and initial value $2 \alpha_{0}$. The fact that the "initial increase" of the sojourn density of Brownian motion at the starting point is a.s. strictly positive leads easily to the representation of $T\left(x_{0}, \alpha_{0}\right)$ as the inf. of $t$ for which the sojourn density exceeds $2 \alpha_{0}$ at $x_{0}$. In the contrary case, one could let $T$ denote the inf. of $t$ for which the derivative for the sojourn density at $x_{0}$, taken only with respect to the sequence of differential quotients with increments $\beta_{k}$, existed and was at least $2 \alpha_{0}$, and $T$ would clearly be a stopping time for $R_{\infty}(t)$ for which, by hypothesis, $P\left\{T<T\left(x_{0}, \alpha_{0}\right)\right\}>0$ would hold. There would then be with positive probability a positive increase in the density during $\left(T, T\left(x_{0}, \alpha_{0}\right)\right)$ in such a way that the density up to time $T\left(x_{0}, \alpha_{0}\right)$ could not be $2 \alpha_{0}$.

To remove the restriction of $x_{0}$ to diadic rationals one may choose $x_{i}$ such that $\left|x_{i}\right|<\varepsilon, x_{i}<0$, and $x_{0}-x_{i}$ is a diadic rational. It is evident from Theorem 2.1 that the maximum of the sojourn density up to the first arrival at $x_{i}$ becomes small with $\varepsilon$ (even if $x_{i}$ is not diadic rational), and the theorem in this case follows by its application to the process following the first arrival at $x_{i}$. The uniformity of convergence near $x=x_{0}$ will follow if it is shown that the maximum of the sojourn density added during the interval between $T(k)$ and $T\left(x_{0}, \alpha_{0}\right)$ becomes small a.s. as $k$ increases. This density is of course a diffusion process of the type considered with initial value $2\left|s(k)-\alpha_{0}\right|$, and the maximum tends to 0 along with the initial value, being a monotone function of it for $s(k) \geqq \alpha_{0}$, and for $s(k)<\alpha_{0}$, considered separately. Lastly, to remove the restriction of $\alpha_{0}$ to diadic rationals, one has only to choose $\alpha_{1, i} \uparrow \alpha_{0}, \alpha_{2, i} \downarrow \alpha_{0}$, where $\alpha_{1, i}$ and $\alpha_{2, i}$ are diadic rationals, and use the now apparent convergence of $T\left(x_{0}, \alpha_{1, i}\right)$ to $T\left(x_{0}, \alpha_{2, i}\right)$. In this way the proof of Theorem 2.2 is concluded for $\alpha_{0}>0$. In the special case 
of $\alpha_{0}=0$, it is evident that $T\left(x_{0}, \alpha_{0}\right)$ becomes the first passage time to $x_{0}$. Theorem 2.2 is then an immediate consequence of Theorem 2.1 with $m=0$, coupled with the preceding remarks to remove the limitation of $x_{0}$ to the diadic rationals.

A disadvantage of Theorem 2.2 from the standpoint of disclosing the general behavior of the sojourn densities is that, except in the trivial case $x_{0}=\alpha_{0}=0$, the expectation of $T\left(x_{0}, \alpha_{0}\right)$ is infinite. The theorem, therefore, does not give very direct information about the densities in short time periods. A better idea is provided by considering the process up until the first arrival in $\left\{ \pm x_{0}\right\}$. This case is easily reduced to the former. Let $T$ denote the first passage time of $R_{\infty}(t)$ to $\{ \pm 1\}$, and consider the conditional distributions of $f(x, T, w)$ given that $R_{\infty}(T)=-1$. For $-1 \leqq x \leqq 1$, since this condition is imposed at an endpoint, it does not destroy the Markovian property, but the conditional process is no longer homogeneous in $-1 \leqq x \leqq 0$ or in $0 \leqq x \leqq 1$. Let $p_{-1}\left(y_{1}, x_{1} ; y_{2}, x_{2}\right)$, $-1 \leqq x_{1}<x_{2} \leqq 1$, denote the conditional transition density of $f(x, T, w)$ given that $R_{\infty}(T)=-1$, where the existence will be shown immediately. Letting $p$ and $P$ denote probability densities and probabilities, respectively, we have $p_{-1}\left(y_{1}, x_{1} ; y_{2}, x_{2}\right)=A B C^{-1}$ where

$$
\begin{aligned}
& A=p\left(f\left(x_{2}, T(-1,0), w\right)=y_{2} \mid f\left(x_{1}, T(-1,0), w\right)=y_{1}\right), \\
& B=P\left(f(1, T(-1,0), w)=0 \mid f\left(x_{2}, T(-1,0), w\right)=y_{2}\right),
\end{aligned}
$$

and

$$
C=P\left(f(1, T(-1,0), w)=0 \mid f\left(x_{1}, T(-1,0), w\right)=y_{1}\right) .
$$

For $0 \leqq x_{1}<x_{2} \leqq 1$, this becomes, by use of Theorem 1.2 and a change of variables corresponding to the factor $\frac{1}{2}$ of Theorem 2.2 ,

$p_{-1}\left(y_{1}, x_{1} ; y_{2}, x_{2}\right)$

$$
\begin{aligned}
=\frac{y_{1}}{2\left(x_{2}-x_{1}\right)^{2}} \exp \left(-\frac{y_{1}+y_{2}}{2\left(x_{2}-x_{1}\right)}-\right. & \left.\frac{y_{2}}{2\left(1-x_{2}\right)}+\frac{y_{1}}{2\left(1-x_{1}\right)}\right) \\
& \cdot \sum_{k=0}^{\infty} \frac{1}{k !(k+1) !}\left(\frac{\sqrt{ }\left(y_{1} y_{2}\right)}{2\left(x_{2}-x_{1}\right)}\right)^{2 k} .
\end{aligned}
$$

For $-1 \leqq x_{1}<x_{2} \leqq 0$ it is necessary to use the transition function corresponding to the generator $4 y d^{2} / d y^{2}+2 d / d y$ of $f(-1+x, T(-1,0), w)$. It was shown in the proof of Corollary 1.3 that the transition function corresponding to $y d^{2} / d y^{2}+d / d y$ (i.e., of $s(-1,0 ; x)$ ) is the convolution of that of Theorem 1.2 and another distribution whose Laplace transform is $\left(\lambda\left(x_{2}-x_{1}\right)+1\right)^{-1}$. The latter distribution evidently has the density $\left(x_{2}-x_{1}\right)^{-1} \exp \left(-y\left(x_{2}-x_{1}\right)^{-1}\right)$, and the required transition function for the process of Corollary 1.3 in $0 \leqq x \leqq-x_{0}$ is found to be 


$$
\begin{gathered}
\left\{\frac{y_{1} y_{2}}{\left(x_{2}-x_{1}\right)^{3}} \exp \left(-\frac{y_{1}+y_{2}}{x_{2}-x_{1}}\right) \sum_{k=0}^{\infty} \frac{1}{(k+1) !^{2}}\left(\frac{\sqrt{ }\left(y_{1} y_{2}\right)}{x_{2}-x_{1}}\right)^{2 k}\right\} \\
+\left(x_{2}-x_{1}\right)^{-1} \exp \left(-\frac{y_{1}+y_{2}}{x_{2}-x_{1}}\right) .
\end{gathered}
$$

To compute the probabilities $P\left(f(1, T(-1,0), w)=0 \mid f\left(x_{i}, T(-1,0), w\right)=y_{i}\right)$, $i=1$ or 2 , for $-1 \leqq x_{1}<x_{2} \leqq 0$, it is only necessary to use this transition function as in the previous case to determine the conditional distribution in $y$ of $f(0, T(-1,0), w)$, multiply by $e^{-y}$, and integrate over $y$. The resulting formulas will not be written down-it is clear that what has been said is sufficient to determine $p_{-1}\left(y_{1}, x_{1} ; y_{2}, x_{2}\right)$ completely. The transition density $p_{1}\left(y_{1}, x_{1} ; y_{2}, x_{2}\right)$ of $f(x, T, w)$ given that $R_{\infty}(T)=1$ is derived from $p_{-1}\left(y_{1}, x_{1} ; y_{2}, x_{2}\right)$ by using the symmetry of the process $R_{\infty}(t)$ about 0 . This leads immediately to the relation $p_{1}\left(y_{1}, x_{1} ; y_{2}, x_{2}\right)=p_{-1}\left(y_{2},-x_{2} ; y_{1},-x_{1}\right) p_{-1}\left(0,-1 ; y_{2},-x_{2}\right) p_{-1}^{-1}\left(0,-1 ; y_{1},-x_{1}\right)$. Since we have $P\left\{R_{\infty}(T)=-1\right\}=P\left\{R_{\infty}(T)=1\right\}=\frac{1}{2}$, the joint distributions of $f(x, T, w),-1 \leqq x \leqq 1$, are determined as $\frac{1}{2}$ times the sum of the corresponding conditional joint distributions, and are thus completely known.

3. The purpose of this section is to derive from Theorem 2.2 the existence and certain properties of the sojourn time densities $f(x, t, w)$ for fixed $t$.

DeFINITION 3.1. Let

$$
f(x, t, w)=\frac{d}{d x} \int_{0}^{t} I_{(-\infty, x)}\left(R_{\infty}(\tau)\right) d \tau
$$

if the derivative exists for all $x$ and $t$, and is continuous in $(x, t)$. Otherwise, set $f(x, t, w)=0$.

THEOREM 3.1 (The THEOREM OF [12]). With probability $1, f(x, t, w)$ exists as a derivative for all $x$ and $t$, and is continuous in $(x, t)$.

The proof of this theorem will be completed following a derivation of an estimate for $\max _{x} f(x, t, w)$. For the present we prove only that, for fixed $t$, the derivative defining $f(x, t, w)$ exists and is continuous in $x,-\infty<x<\infty$, a.s. We shall use the known fact that the derivative defining $f(0, t, w)$ exist a.s. $[12 ; 14]$, although this also can be derived by the present methods (see $\$ 4$ ). Let us set

$$
S(t, \alpha)=\{w: t<T(0, \alpha)\}
$$

(from Theorem 2.2). Then $S(t, \alpha)$ is nondecreasing in $\alpha$, and since it is easily seen from the proof of Theorem 2.2 that $\lim _{\alpha \rightarrow \infty} T(0, \alpha)=\infty$ a.s., it is clear that $\lim _{\alpha \rightarrow \infty} P(S(t, \alpha))=1$. Suppose that $R_{\infty}(t)$ and $f(0, t, w)$ are given, and let $s^{*}\left(x_{0}, \alpha_{0} ; x\right)$ be the replica of $s\left(x_{0}, \alpha_{0} ; x\right)$ generated by the conditional Brownian motion process $R_{\infty}(t+\tau)-R_{\infty}(t), \tau \geqq 0$. Theorem 2.2 implies that 
$s^{*}\left(-R_{\infty}(t), \alpha-\frac{1}{2} f(0, t, w) ; x-R_{\propto}(t)\right)$ on $S(t, \alpha)$ exists, is $\frac{1}{2}$ of a sojourn density, and is continuous in $x$ a.s. with respect to the conditional probability. Therefore the same process exists and is continuous in $x$ unconditionally a.s. on $S(t, \alpha)$, when $R_{\infty}(t)$ and $f(0, t, w)$ are determined by $R_{\infty}(\tau), 0 \leqq \tau \leqq t$. Hence there also exists on $S(t, \alpha)$ the process

$$
\frac{1}{2} f(x, t, w)=s(0, \alpha ; x)-s^{*}\left(-R_{\infty}(t), \alpha-\frac{1}{2} f(0, t, w) ; x-R_{\infty}(t)\right)
$$

and it is continuous in $x$ a.s. Letting $\alpha$ become large, the assertion follows.

The next aim is to estimate the distibution of $\max _{x} f(x, t, w)$. Since we are concerned only to show that the probabilities of large values are sufficiently small, it will be enough to make the estimate under the condition $R_{\infty}(t)=0$, it being evident that this conditioning increases $P\left\{\max _{x} f(x, t, w)>y\right\}$ for each $y>0$ (see note 6 below). It will also be assumed at first that $\alpha=\frac{1}{2} f(0, t, w)$ is given. Defining the conditional probabilities by a method used in [12], the quantity to be estimated equals $\lim _{\Delta \rightarrow 0} P\left(\max _{x} f(x, t, w)>y \mid\right.$ for some $t^{\prime} \in(t, t+\Delta), R_{\infty}\left(t^{\prime}\right)=0$ and $\left.\frac{1}{2} f\left(0, t^{\prime}, w\right)=\alpha\right)$. We first show that $P\left(T(0, \alpha)=t \mid \frac{1}{2} f(0, t, w)=\alpha\right.$ and $\left.R_{\infty}(t)=0\right)=1$. It has been seen in the proof of Theorem 2.2 that $P\left\{\frac{1}{2} f(0, t, w)=\alpha\right.$ for some $t>T(0, \alpha)\}=0$, and therefore, $P$ (for some $t^{\prime \prime}<t, \frac{1}{2} f\left(0, t^{\prime \prime}, w\right)=\alpha \mid$ for some $t^{\prime} \in(t, t+\Delta), R_{\infty}\left(t^{\prime}\right)=0$ and $\left.\frac{1}{2} f\left(0, t^{\prime}, w\right)=\alpha\right)=0$. Letting $\Delta$ approach 0 , the assertion is evident. It is now clear that if $s(0, \alpha ; x)$ is considered as a function of $x,-\infty<x<\infty$, as suggested in the proof of Theorem 2.2, then one has the relations

$$
\begin{aligned}
P\left(\max _{x} f(x, t, w)\right. & \left.>y \mid R_{\infty}(t)=0 \text { and } \frac{1}{2} f(0, t, w)=\alpha\right) \\
& =P\left(\max _{x} 2 s(0, \alpha ; x)>y \mid \int_{-\infty}^{\infty} 2 s(0, \alpha ; x) d x=t\right) \\
& =\lim _{\Delta \rightarrow 0} P\left(\max _{x} 2 s(0, \alpha ; x)>y \mid \int_{-\infty}^{\infty} 2 s(0, \alpha ; x) d x \in(t, t+\Delta)\right) \text { a.s. }
\end{aligned}
$$

Let $M(\alpha)$ denote $\max _{x \geqq 0} 2 s(0, \alpha ; x)$, and $I(\alpha)$ denote $\int_{-\infty}^{\infty} 2 s(0, \alpha ; x) d x$, so that the last term above is bounded by

$$
\begin{aligned}
2 P(M(\alpha)> & y \mid I(\alpha)=t) \\
& =\lim _{\Delta \rightarrow 0} 2 P(I(\alpha) \in(t, t+\Delta) \mid M(\alpha)>y) P\{M(\alpha)>y\} P^{-1}\{I(\alpha) \in(t, t+\Delta)\} .
\end{aligned}
$$

In the first place, since the process $s(0, \alpha ; x)$ has no drift, comparison with a Brownian motion process immediately shows that $P\{M(\alpha)>y\}=\alpha y^{-1}$. We do not propose to determine the ratio of the remaining two probabilities exactly, but merely observe that if $(t, t+\Delta)$ were replaced by $(t, 2 t)$ for $\Delta<t$ the ratio 
would be increased. Using the known distribution of $f(0, t, w)$ [Lévy, 12, (39), p. 316] one has

$$
\begin{aligned}
P\{I(\alpha) \in(t, 2 t)\} & =P\{f(0, t, w)<2 \alpha\}-P\{f(0,2 t, w)<2 \alpha\} \\
& =\sqrt{\frac{2}{\pi t}} \int_{\sqrt{ } 2 \alpha}^{2 \alpha} \exp \left(-\frac{x^{2}}{2 t}\right) d x .
\end{aligned}
$$

As concerns $P(I(\alpha) \in(t, 2 t) \mid M(\alpha)>y)$, one can derive a bound by comparing $s(0, \alpha ; x), x \geqq 0$, with a Brownian motion and using the reflection principle argument of Désiré André. Under the supposition that $M(\alpha)>y$ and $I(\alpha)<2 t$ hold, there must be a passage from $y / 2$ to $y / 4$ by the process $s(0, \alpha ; x)$ within a parameter interval of length $4 t y^{-1}$ "following" the first arrival at $y / 2$. Since the infinitesimal generator of $s(0, \alpha ; x)$ is $z d^{2} / d z^{2}=(d / d m) d / d z$, where $m(z)$ $=\ln (z),[6]$, it can be seen from the interpretation of $m(z)$ as a "speed measure", the speed of the process increasing at larger values, that the probability of such a passage is less than it would be for a passage from $y / 2$ to $3 y / 4$ in the same interval, which in turn is less than that of the same passage for the process with generator $(3 y / 4) d^{2} / d z^{2}$. This process can be written as $X(3 y t / 2)$ where $X(t)$ is Brownian motion, and its probability of reaching $3 y / 4$ from $y / 2$ before time $4 t y^{-1}$ is $1 / \sqrt{ }(3 \pi t) \int_{y / 4}^{\infty} \exp \left(-x^{2} / 12 t\right) d x$. Combining all of these estimates, we find that

$$
\begin{aligned}
P\left\{\max _{x} f(x, t, w)>y\right\} & <2 P\left(\max _{x \geqq 0} f(x, t, w)>y \mid R_{\infty}(t)=0\right) \\
& <2 E\left[\frac{\alpha \int_{y / 4 \sqrt{ }(6 t)}^{\infty} e^{-x^{2} / 2} d x}{y \int_{2 \alpha / \sqrt{ }(2 t)}^{2 \alpha / \sqrt{ }} e^{-x^{2} / 2} d x}\right],
\end{aligned}
$$

where the expectation is over $\alpha$ and must be taken with respect to the conditional distribution of $2^{-1} f(0, t, w)$ given that $R_{\infty}(t)=0$. This distribution has been found by $P$. Lévy, who showed that

$$
P\left(f(0, t, w)>x \mid R_{\infty}(t)=0\right)=\exp -x^{2}(2 t)^{-1}
$$

([12, p. 316], where $f(0, t, w)$ has the same distribution as " $\sqrt{ }(\pi / 2) S(t)$ ", as is also shown there). If we estimate the ratio of the terms involving $\alpha$ in (3.1) by separating the integral into parts corresponding to the intervals $(0, \sqrt{ } t)$ and $(\sqrt{ } t, \infty)$, replacing the denominator by the smaller quantity

$$
\alpha t^{-1 / 2}(2-\sqrt{ } 2) \exp \left(-2 \alpha^{2} t^{-1}\right)
$$

in $(0, \sqrt{ } t)$, and using the standard inequalities for the normal distribution [7, p. $166]$ to bound it from below by $\sqrt{ }(2 t) 4^{-1} \exp \left(-\alpha^{2} t^{-1}\right)$ in $(\sqrt{ } t, \infty)$, it follows that this expectation is less than $c \sqrt{ } t$ for a constant $c<10$. Applying the other half of 
the normal inequalities to the resulting function of $y$ in (3.1) we find that for a constant $K<200$,

$$
P\left\{\max _{x} f(x, t, w)>y\right\}<K t y^{-2} \exp \left(-\left(y^{2}(192 t)^{-1}\right)\right) .
$$

This is the required estimate.

The proof of Theorem 3.1 is now completed without difficulty. Choosing a large number $B$, we subdivide $(0, B)$ into subintervals of length $B / n$, and note that

$$
\begin{aligned}
\sum_{i=1}^{n} P\left\{\operatorname { m a x } _ { x } \left(f\left(x, \frac{i B}{n}, w\right)-\right.\right. & \left.\left.f\left(x, \frac{(i-1) B}{n}, w\right)\right)>y\right\} \\
& =n P\left\{\max _{x} f\left(x, \frac{B}{n}, w\right)>y\right\}<\frac{K B^{2}}{n y^{2}} \exp \left(-\frac{n y^{2}}{192 B}\right) .
\end{aligned}
$$

As $n$ becomes large this approaches 0 , and it is evident that if $S$ is a countable dense set in $(0, B)$ then since the existence and continuity in $x$ of $f(x, t, w)$ for $t \in S$ a.s. may be assumed, $f(x, t, w)$ is a.s. both jointly continuous in $x$ and $t \in S$ and continuous in $t \in S$ uniformly in $x$. Let it be assumed, for purposes of showing that the corresponding event has probability 0 , that $\left(x_{0}, t_{0}, w\right)$ is a point at which either the derivative $f(x, t, w)$ is not defined, or at which $f(x, t, w)$ is defined but not continuous in $(x, t)$. In the first instance there exist sequences of sojourndensity-approximating differential quotients approaching either $\infty$ or two different values at $\left(x_{0}, t_{0}\right)$. By choosing $t_{1}<t_{0}<t_{2} ; t_{1}, t_{2} \in S ; t_{2}-t_{1}$ small, and considering the corresponding quotients at $\left(x_{0}, t_{i}, w\right), i=1$ or 2 , using the monotonicity of each quotient in $t$ and the continuity of their limits (for fixed $t$ in $S$ ) a.s., it seen that the first instance represents a set of probability 0 . In the second instance, having established the existence of $f(x, t, w)$, one selects $t_{1}$ and $t_{2}$ as before and observes that outside of a set of probability 0 one has $f\left(x, t_{1}, w\right) \leqq f(x, t, w) \leqq f\left(x, t_{2}, w\right)$ while $f\left(x, t_{2}, w\right)-f\left(x, t_{1}, w\right)<\varepsilon$ and both terms are continuous in $x$. It follows from this that $f(x, t, w)$ is continuous in $(x, t)$, and the proof of Theorem 3.1 is complete.

4. In this section attention is returned to the random walks $R_{k}\left(n \alpha_{k}\right)$, and the convergence of their discrete "sojourn density processes" to $f(x, t, w)$ on $\left(\Omega_{\infty}, F_{\infty}, P_{\infty}\right)$.

(6) A further remark concerning the use of the hypothesis that $R_{\infty}(t)=0$ is in order, since no proof has been indicated for the assertion that an upper bound for $P\left\{\max _{x} f(x, t, w)>y\right\}$ derived under this hypothesis is also an unconditional upper bound (although this is clear intuitively). It is possible, in fact, to carry out estimates analogous to those given here but under the condition that $R_{\infty}(t)=b$, and then take an expectation in $b$. Using a formula of Lévy for the conditional distribution of the first passage time to $b$ given that $R_{\infty}(t)=b[12,(43)$, p. 318] it follows that for each $b$ the estimate (3.2) remains valid, and, therefore is unaffected by the expectation over $b$. 
Definition 4.1. Let $f_{k}(x, t, w)=2 \beta_{k} S(t, i)$ for $i \beta_{k} \leqq x<(i+1) \beta_{k}$, where $S(t, i)$ is the number of steps of $R_{k}$ from $i \beta_{k}$ to $(i+1) \beta_{k}$ in the first $\left[\alpha_{k}^{-1} t\right]$ steps.

What is to be shown is that $f_{k}(x, t, w)$ converges to $f(x, t, w)$. It is first necessary to transfer the estimate of $\max _{x} f(x, t, w)$ to an estimate of $\max _{x} f_{k}(x, t, w)$.

LEMMA 4.1. For $k>K(t)$ sufficiently large,

$$
P\left\{\max _{x} f_{k}(x, t, w)>y\right\}<K_{1} t y^{-2} \exp \left(-\left(y^{2}\left(K_{2} t\right)^{-1}\right)\right)
$$

where $K_{1}$ and $K_{2}$ are fixed constants.

Proof. It is obviously sufficient to prove the lemma for $t$ of of the form $n \alpha_{k}$. Let the quantities $R_{k}\left(m \alpha_{k}\right), 0 \leqq m \leqq n$, be given, and for $r \geqq k$ let $s_{k, r}(x, t, w)$ denote $\beta_{r}\left(S^{-}(k, r, t, i)+S^{+}(k, r, t, i)\right)$ for $i \beta_{r} \leqq x<(i+1) \beta_{r}$, where $S^{ \pm}(k, r, t, i)$ are, respectively, the numbers of steps of $R_{r}$ from $i \beta_{r}$ to $(i \pm 1) \beta_{r}$ up to the time when $R_{k}$ completes its first $n$ steps. Under these conditions it is clear that, for $x$ of the form $i \beta_{k}, s_{k, r}(x, t, w)$ is a martingale in $r \geqq k$, and the martingale convergence theorem applies to yield that $\lim _{r \rightarrow \infty} s_{k, r}(x, t, w)$ exists a.s. In fact, $s_{k, r}$ is directly analogous to $M_{k_{1}, k_{2}}$ in the proof of Theorem 2.2. Let $x=i \beta_{k}$ be a point at which $f_{k}(x, t, w)$ attains its maximum, and let $T$ be the time at which $R_{\infty}(t)$ completes the $n$th consecutive disjoint passage of a distance $\beta_{k}$. It will be shown that with (conditional) probability at least $\frac{1}{4}$, for $k>K(t)$ sufficiently large, the inequalities

$$
f_{k}(x, t, w)<3 f(x, T, w)<3 f(x, t, w)
$$

hold. From this result and (3.2) the lemma follows immediately.

It is not hard to see, first of all, that $\lim _{r \rightarrow \infty} s_{k, r}(x, t, w)=f(x, T, w)$ a.s. This becomes apparent upon noting first that for $x=i \beta_{k}$ one has as in the proof of Lemma 2.2 (or by the law of large numbers)

$$
\lim _{r \rightarrow \infty} s_{k, r}(x, t, w)=\lim _{r \rightarrow \infty} 2 \beta_{r} S^{+}\left(k, r, t, x \beta_{r}^{-1}\right) \text { a.s. }
$$

For each fixed $m^{*}$ and $b$ such that $(b+1) \beta_{k} \leqq x$, Theorem 2.2 implies that

$$
\lim _{r \rightarrow \infty} 2 \beta_{r} S\left(k, r, b, m^{*}, x \beta_{r}^{-1}\right)=f\left(x, T\left(k, b, m^{*}\right), w\right) \text { a.s. }
$$

(see Definitions 2.1 and 2.3). When $R_{k}\left(m \alpha_{k}\right), 0 \leqq m \leqq n$, are given, then one sees as in Theorem 2.1 that

$$
\begin{aligned}
& \lim _{r \rightarrow \infty} 2 \beta_{r}\left(S\left(k, r, b, m^{*}, x \beta_{r}^{-1}\right)-S^{+}\left(k, r, t, x \beta_{r}^{-1}\right)\right) \\
& \quad=f\left(x, T\left(k, b, m^{*}\right), w\right)-f(x, T, w) \text { a.s. on the set }\left\{T\left(k, b, m^{*}\right)>T\right\},
\end{aligned}
$$

which is determined by the given data, and that this limit is independent of $\lim _{r \rightarrow \infty} 2 \beta_{r} S^{+}\left(k, r, t, x \beta_{r}^{-1}\right)$. Letting $m^{*}$ become large, the assertion follows. By the proof of Theorem 2.2, $\mathrm{s}_{k, r}(x, t, w)$ has (conditional) variance less than 
$\frac{1}{2} \beta_{k} s_{k, k}(x, t, w)$ and the same, therefore, holds for $f(x, T, w)$. The Tchebycheff inequality implies that

$$
P\left\{\left|f(x, T, w)-s_{k, k}(x, t, w)\right|>\varepsilon\right\}<\frac{\beta_{k}^{2} s_{k, k}^{2}(x, t, w)}{4 \varepsilon^{2}},
$$

and, therefore, setting $\varepsilon=\frac{2}{3} s_{k, k}(x, t, w)$ when $k$ is large, the inequalities $f_{k}(x, t, w)<2 s_{k, k}(x, t, w)<3 f(x, T, w)$ hold with probability near to 1 . Finally, letting $n$ and $k$ increase with $t$ fixed, the central limit theorem shows that $T$ (depending on $(n, k))$ regarded as a sum of $n$ (conditionally) independent random variables (1st passage times), is asymptotically normally distributed with mean $t$, and hence that $P\{T<t\}>\frac{1}{4}$ holds for large $k$. The inequalities (4.1) are therefore proved, and the lemma follows.

Using this lemma, we are in a position to prove the intended theorem

THEOREM 4.1. With probability $1, \lim _{k \rightarrow \infty} f_{k}(x, t, w)=f(x, t, w)$ uniformly in $(x, t)$ for $t$ in any finite interval.

Proof. The method of proof is as follows. We assume that for a large constant $C$ it is given that $\max _{x} f_{k}(x, t, w)<C$, where $t$ has the form $n \alpha_{k}$, and consider for $x$ of the form $m \beta_{k}$ the total sojourn time period of $R_{\infty}$ during steps of $R_{k}$ from $x$ to $\left\{x \pm \beta_{k}\right\}$ in the first $n$ steps, which we denote by $S_{k}(x, t, w)$. The main point is then to estimate

$$
P\left\{\max _{x}\left|s_{k, k}(x, t, w)-\beta_{k}^{-1} S_{k}(x, t, w)\right|>\varepsilon\right\} .
$$

It is shown that this is sufficiently small to conclude, after using Lemma 4.1 to remove the conditioning by $C$, that as $k$ increases $\max _{x}\left|s_{k, k}(x, t, w)-\beta_{k}^{-1} S_{k}(x, t, w)\right|$ converges to 0 a.s. It is not very hard to realize that $s_{k, k}(x, t, w)-f_{k}(x, t, w)$ converges to 0 and that $\beta_{k}^{-1} S_{k}(x, t, w)$ converges to $f(x, t, w)$, from which the theorem follows.

Turning to the details, let it be given that $\max _{x} f_{k}(x, t, w)<C$ (for a $t$ of the form $n \alpha_{k}$ ) so that, for $x$ of the form $m \beta_{k}, s_{k, k}(x, t, w)<C+\beta_{k}$ holds. The essential observation is that the distribution of $s_{k, k}(x, t, w)-\beta_{k}^{-1} S_{k}(x, t, w)$ is identical with that of $\beta_{k}^{-1} \lim _{r \rightarrow \infty} L_{k, r}\left(\beta_{k} s_{k, k}(x, t, w)\right)$ where $L_{k, r}(\cdot)$ is the "time lag'" between $R_{k}$ and $R_{r}$, as defined in [10], and where $L_{k, r}(\cdot)$ and $s_{k, k}(x, t, w)$ are independent. This fact is clear from the definition of $L_{k, r}(\cdot)$. To show that is small enough, however, it is not sufficient to use the methods of [10], based only on the variance of $L_{k, r}$. Instead we shall need the central limit theorem for large deviations [Cramer, 2]. To justify this application we note first that $\lim _{r \rightarrow \infty} L_{k, r}\left(\beta_{k} s_{k, k}(x, t, w)\right.$ is (conditionally) a sum of $\beta_{k}^{-1} s_{k, k}(x, t, w)$ independent and identically distributed random variables with mean 0 and variance less than $\frac{2}{3} \alpha_{k}^{2}$ - a fact used above in another context. It must be shown that for some $a>0$ these variables 
satisfy Cramer's condition $E e^{a|X|}<\infty$. But since they are merely the 1 st passage times of $R_{\infty}$ from $x$ to $\left\{x \pm \beta_{k}\right\}$, centered at their expectation, their exact distribution is well known [11], and the condition is obviously met. The theorem on large deviations now yields that for $\varepsilon>0, \varepsilon\left(\beta_{k} s_{k, k}(x, t, w)\right)^{-1 / 2}<\beta_{k}^{-\alpha} s_{k, k}(x, t, w)^{\alpha}$, and $0<\alpha<1 / 6$,

$$
P\left\{\left|s_{k, k}(x, t, w)-\beta_{k}^{-1} S_{k}(x, t, w)\right|>\varepsilon\right\}<1-\phi\left(\varepsilon\left(\beta_{k} s_{k, k}(x, t, w)\right)^{-1 / 2}\right)
$$

where $\phi$ is the normal distribution. Under our hypotheses this probability would only be increased by setting $s_{k, k}(x, t, w)=C+\beta_{k}$, which yields the conclusion that for $C_{k} \geqq \beta_{k}^{-\gamma}, \gamma>(1-2 \alpha)(1+2 \alpha)^{-1}$,

$$
\begin{aligned}
P\left\{\left|s_{k, k}(x, t, w)-\beta_{k}^{-1} S_{k}(x, t, w)\right|\right. & >\varepsilon\} \\
& <\frac{\beta_{k}\left(C+\beta_{k}\right)^{1 / 2}}{\varepsilon \sqrt{ }(2 \pi)} \exp \left(-\left(\varepsilon^{2}\left(2 \beta_{k}\left(C_{k}+\beta_{k}\right)\right)^{-1}\right)\right) .
\end{aligned}
$$

The only values of $x$ for which $s_{k, k}(x, t, w)$ does not vanish are those for which $|x| \leqq \max _{0 \leqq \tau \leqq t}\left|R_{k}(\tau)\right|$ holds, and for this maximum the usual reflection principle argument shows that for $N$ large

$$
P\{\max >N\}<\frac{4 \sqrt{ } t}{N \sqrt{ }(2 \pi)}-\exp \left(-\frac{1}{2} N^{2} t^{-1}\right) .
$$

We now set $\alpha=\frac{1}{8}, N_{k}=\beta_{k}^{-1 / 2}$ and $C_{k}=t^{1 / 2} \beta_{k}^{-3 / 5}$. Combining (4.2), (4.3), and Lemma 4.1 , it follows by considering only $-N_{k} \leqq x \leqq N_{k}$ that

$$
\begin{aligned}
P\left\{\max _{x, k \geqq k_{1}}\left|s_{k, k}(x, t, w)-\beta_{k}^{-1} S_{k}(x, t, w)\right|>\varepsilon\right\} \\
<\sum_{k=k_{1}}^{\infty}\left[\frac{2 \beta_{k}^{-1 / 2}\left(t^{1 / 2} \beta_{k}^{-3 / 5}+\beta_{k}\right)}{\varepsilon \sqrt{ }(2 \pi)} \exp \left(-\left(\varepsilon^{2}\left(2 \beta_{k}\left(t^{1 / 2} \beta_{k}^{-3 / 5}+\beta_{k}\right)\right)^{-1}\right)\right)\right. \\
\left.+\frac{4\left(t \beta_{k}\right)^{1 / 2}}{\sqrt{ }(2 \pi)} \exp \left(-\frac{1}{2}\left(\beta_{k} t\right)^{-1}\right)+2 K_{1} \beta_{k}{ }^{6 / 5} \exp -\left(K_{2}^{-1} \beta_{k}^{-6 / 5}\right)\right] .
\end{aligned}
$$

The sum is clearly finite, proving that for $t$ fixed $s_{k, k}(x, t, w)$ converges uniformly to $\beta_{k}^{-1} S_{k}(x, t, w)$. The restriction on $t$ can be removed by noting that if each summand in (4.4) is multiplied by $\left[t \alpha_{k}^{-1}\right]$, corresponding to the number of discrete values of $t^{\prime}<t$ which need to be considered, the result remains finite. A more natural method, however, is to use the fact that, for $t^{\prime}=m \alpha_{k}$, $s_{k, k}(x, t, w)-\beta_{k}^{-1} S_{k}(x, t, w)$ is a martingale in $m$, as is clear from [10] (or directly). For large $k$ the variance is small for $m \leqq t \alpha_{k}^{-1}$, and the extension of conver- 
gence to all $t^{\prime}=m \alpha_{k} \leqq t$ is completed by a conditional "reductio ad absurdum" in the standard way. It has thus been established that for each $t$

$$
\lim _{k \rightarrow \infty} \max _{x, t^{\prime}=m \alpha_{k}<t}\left|s_{k, k}\left(x, t^{\prime}, w\right)-\beta_{k}^{-1} S_{k}\left(x, t^{\prime}, w\right)\right|=0
$$

a.s. It remains to prove the analogous statements for $\left|s_{k, k}(x, t, w)-f_{k}(x, t, w)\right|$ and for $\left|\beta_{k}^{-1} S_{k}(x, t, w)-f(x, t, w)\right|$. Using the central limit theorem for Bernoulli trials (DeMoivre-Laplace) one finds that for large $k$ and $D>0$,

$$
\left.\left.P\left(\mid s_{k, k}\right) x, t, w\right)-f_{k}(x, t, w)|>\varepsilon| s_{k, k}(x, t, w)=D\right) \cong 1-\phi\left(\varepsilon\left(D \beta_{k}\right)^{-1 / 2}\right),
$$

$\phi$ being the normal distribution. Combining this with the bound of Lemma 4.1 and the fact that $s_{k, k}(x, t, w)-f_{k}(x, t, w)$ is a martingale in $m$ for $t=m \alpha_{k}$, the first statement is immediately evident as in the previous case. The second statement requires more delicate treatment, because the time spent in $\left(x-\beta_{k}, x+\beta_{k}\right)$ during passages of $R_{\infty}(t)$ from $x \pm \beta_{k}$ "back to $x$ " is not included in $S_{k}(x, t, w)$ and these periods have a somewhat different distribution than those during passages from $x$ to $\left\{x \pm \beta_{k}\right\}$, which are entirely spent in $\left(x-\beta_{k}, x+\beta_{k}\right)$. One is led to consider the passages from $x$ to $x+\beta_{k}$ and from $x+\beta_{k}$ back to $x$ only, treating those from $x$ to $x-\beta_{k}$ separately by the same method. Let $Z_{k}^{+}(x, t, w)$ denote the sojourn period of $R_{\infty}$ in $\left(x, x+\beta_{k}\right)$ before time $t$ during the successive disjoint passages from $x$ to $x+\beta_{k}$, where from $x$ immediately upon reaching $x+\beta_{k}$ the process begins a "passage from $x+\beta_{k}$ back to $x$ " (thereupon, after reaching $x$, beginning a new passage from $x$ to $x+\beta_{k}$, etc.) and let $Z_{k}^{-}(x, t, w)$ be the analogous period during passages from $x+\beta_{k}$ back to $x$. Also, let $T(n, x)$ be the time of the $n$th return to $x$ from $x+\beta_{k}$ in this alternation. Then $Z_{k}^{+}(x, T(n, x), w)-Z_{k}^{-}(x, T(n, x), w)$ is a sum of $n$ independent and identically distributed symmetric random variables each with variance less than $4 \alpha_{k}^{2} / 3$. Since the number of passages from $x$ to $x+\beta_{k}$ by time $T(n, x)$ is equal to $n$, Lemma 4.1 and [10] show that for $t$ of the form $n \alpha_{k}, \alpha_{k}^{-1} \leqq n$,

$$
\begin{aligned}
P\left\{\min _{x} T\left(\alpha_{k}^{-1}, x\right)<\lim _{r \rightarrow \infty} L_{k, r}(t)\right\} & =P\left\{\max _{x} f_{k}(x, t, w)>2 \beta_{k}^{-1}\right\} \\
& <\left(K_{1} / 4\right) t \beta_{k}^{2} \exp \left(-\left(4 \beta_{k}^{-2}\left(K_{2} t\right)^{-1}\right)\right) .
\end{aligned}
$$

Combining this with Tchebycheff's inequality and [10, Theorem 2] we find that for large $k$ and $\delta>0$,

$$
P\left\{\max _{x} \beta_{k}^{-1}\left|Z_{k}^{+}(x, t-\delta, w)-Z_{k}^{-}(x, t-\delta, w)\right|>\varepsilon\right\}<A+\frac{4 \alpha_{k}}{3 \varepsilon^{2}}+\frac{2 t \alpha_{k}}{3 \delta^{2}},
$$

where $A$ is the last expression in (4.5). Since the distributions of $Z_{k}^{+}-Z_{k}^{-}$ are symmetric, the same inequality holds uniformly in $\tau=m \alpha_{k} \leqq t-\delta$ after multiplication by 2 on the right. Summing over $k$ the result is finite, implying that for all $t$, 


$$
\lim _{k \rightarrow \infty} \max _{x, t^{\prime}=m \alpha_{k} \leqq t} \beta_{k}^{-1}\left|Z_{k}^{+}\left(x, t^{\prime}, w\right)-Z_{k}^{-}\left(x, t^{\prime}, w\right)\right|=0 \text { a.s. }
$$

From the uniform convergence of the time lag $L_{k, r}(t)$ to 0 a.s. when $k$ and $r$ become large and $0 \leqq t^{\prime} \leqq t$, which is proved in [10], one readily sees that the time of the $\left(\alpha_{k}^{-1} t^{\prime}\right)$ th step of $R_{k}$, which figures in the definition of $S_{k}\left(x, t^{\prime}, w\right)$, converges uniformly to $t^{\prime}, 0 \leqq t^{\prime} \leqq t$, and from the continuity of $f(x, t, w)$ it is clear that the quantity $\beta_{k}^{-1}\left(Z_{k}^{+}\left(x, t^{\prime}, w\right)+Z_{k}^{-}\left(x-\beta_{k}, t^{\prime}, w\right)\right)$ converges uniformly to $\beta_{k}^{-1} S_{k}\left(x, t^{\prime}, w\right)$ a.s., $-\infty<x<\infty, 0 \leqq t^{\prime} \leqq t$. By the same continuity, the quantity $\left(2 \beta_{k}\right)^{-1}\left(Z_{k}^{+}\left(x, t^{\prime}, w\right)+Z_{k}^{+}\left(x-\beta_{k}, t^{\prime}, w\right)+Z_{k}^{-}\left(x, t^{\prime}, w\right)+Z_{k}^{-}\left(x-\beta_{k}, t^{\prime}, w\right)\right)$ converges uniformly a.s. to $f\left(x, t^{\prime}, w\right)$. By (4.7) it is evident that the second quantity is uniformly near the first when $k$ is large, and the proof is complete for $t$ of the form $n^{*} \alpha_{k}$ (for all $k$ ). But this obviously implies that the theorem holds as stated.

By application of Theorem 4.1 we shall construct all diffusion processes on the real line in an interval of their regularity, and with absorbing barriers as endpoints (in the case of accessible endpoints). If the interval is $(\alpha, \beta)$, $-\infty \leqq \alpha<0<\beta \leqq+\infty$, as may be assumed, then after a change of scale the process is determined by an infinitesimal generator in the form of a generalized differential operator $(d / d m)(d / d x)$, [6], operating in an appropriate space of continuous functions on the image $(a, b)$ of $(\alpha, \beta),-\infty \leqq a<0<b \leqq \infty$. Here $m(x)$ is a strictly increasing right continuous function on the interval $(a, b)$, and conversely, any such function gives rise to a unique diffusion process on $(a, b)$ with no "drift" and with absorbing barriers at the accessible endpoints.

The construction could be based directly on the functions $f_{k}(x, t, w)$, but it is more natural to replace them by functions $h_{k}(x, t, w)$ defined as follows:

Definition 4.2. Let $h_{k}(x, t, w)=\beta_{k}(S(t, i)+Q(t, i)), i \beta_{k} \leqq x<(i+1) \beta_{k}$, where $Q(t, i)$ is the number of steps of $R_{k}$ from $(i+1) \beta_{k}$ to $i \beta_{k}$ in the first $\left[\alpha_{k}^{-1} t\right]$ steps, and $S(t, i)$ is introduced in Definition 4.1.

Since $\left|h_{k}(x, t, w)-f_{k}(x, t, w)\right| \leqq \beta_{k}$ holds without exception, it is clear that Theorem 4.1 applies essentially to $h_{k}(x, t, w)$. For a given function $m(x)$ of the type considered, let there be introduced the "time parameters"

and

$$
T_{k}(t)=2^{-1} \int_{a}^{b} h_{k}(x, t, w) d m(x)
$$

$$
T_{\infty}(t)=2^{-1} \int_{a}^{b} f(x, t, w) d m(x) .
$$

Further, let $T$ denote $\inf _{t}: R_{\infty}(t) \notin(a, b)$, where $T=\infty$ is permitted. It is evident from Theorem 4.1 and the continuity of $R_{\infty}(t)$ that the $\operatorname{limit}_{\lim _{k \rightarrow \infty}} T_{k}(t)=T_{\infty}(t)$ holds a.s., for all $t$ in $[0, T)$ and uniformly in $[0, c]$ for all $c<T$, and that $T_{\infty}(t)$ is a monotone continuous mapping of $[0, T)$ onto $\left[0, T_{\infty}(T)\right)$. It follows from 
the uniform convergence of $R_{k}$ to $R_{\infty}$ that $\lim _{k \rightarrow \infty} R_{k}\left(T_{k}(t)\right)=R_{\infty}\left(T_{\infty}(t)\right)$ for all $t$ in $[0, T)$ a.s., uniformly in $[0, c\lrcorner$ for all $c<T$. According to a theorem of Ito and McKean [8], the process $R_{\infty}^{*}\left(t^{*}\right)$ defined by

$$
R_{\infty}^{*}\left(t^{*}\right)=R_{\infty}(t) \text { for } t^{*}=T_{\infty}(t), 0<t^{*}<T_{\infty}(T)
$$

if such $t$ exist; 0 otherwise, is the required diffusion process with initial value 0 , generator $(d / d m)(d / d x)$, and an absorbing barrier at each accessible endpoint. In order to derive $R_{\infty}^{*}\left(t^{*}\right)$ as a uniform limit of processes $R_{k}^{*}\left(t^{*}\right)$ depending only on the random walks $R_{k}$, define $R_{k}^{*}\left(t^{*}\right)=R_{k}\left(n \alpha_{k}\right)$ where $n$ is determined by the inequalities $T_{k}\left(n \alpha_{k}\right) \leqq t^{*}<T_{k}\left((n+1) \alpha_{k}\right)$. Then from the assumption $t^{*}=T_{\infty}(t)$ follow the inequalities $T_{k}\left(n(t) \alpha_{k}\right) \leqq T_{\infty}(t)<T_{k}\left((n(t)+1) \alpha_{k}\right)$, and since $T_{\infty}(t)$ is strictly increasing it is easily seen that $\lim _{k \rightarrow \infty}\left(n(t) \alpha_{k}-t\right)=0$ a.s. for all $t<T$, and consequently, that $\lim _{k \rightarrow \infty} R_{k}^{*}\left(t^{*}\right)=R_{\infty}^{*}\left(t^{*}\right)$ a.s., for all $t^{*}<T_{\infty}(T)$ and uniformly in $[0, c]$ for $c<T_{\infty}(T)$.

A final consideration is warranted for the sojourn density processes corresponding to $R_{\infty}^{*}\left(t^{*}\right)$. In order to avoid irrelevant complication, we shall assume that $a=-\infty$, and $b=+\infty$, which implies that both endpoints are inaccessible. Then it is clear from the definition of $T_{\infty}(t)$ that $T_{\infty}(T)=\infty$, and that for $t \geqq 0$ and all $y$ a.s.

$$
\int_{0}^{T_{\infty}(t)} I_{(-\infty, y)}\left(R_{\infty}^{*}\left(t^{*}\right)\right) d t^{*}=2^{-1} \int_{-\infty}^{y} f(x, t, w) d m(x) .
$$

The sojourn density processes of $R_{\infty}^{*}\left(t^{*}\right)$ are therefore identical with those of $R_{\infty}(t)$, but their definition is obtained by differentiating with respect to the measure $2^{-1} d m(x)$. More precisely, if $\alpha_{0} \geqq 0$ and $x_{0} \leqq 0$ are fixed, and if we set

$$
\left.T\left(x_{0}, \alpha_{0}\right)=\inf _{t^{*}}: \frac{d}{d m\left(x_{0}\right)} \int_{0}^{t^{*}} I_{(-\infty, x)} R_{\infty}^{*}(\tau)\right) d \tau>\alpha_{0},
$$

the process

$$
\left.s\left(x_{0}, \alpha_{0} ; x\right)=\frac{d}{d m(x)} \int_{0}^{T\left(x_{0}, \alpha_{0}\right)} I_{(-\infty, x)} R_{\infty}^{*}(\tau)\right) d \tau
$$

is identical with that of Theorem 2.2 a.s. For a fixed $t$, however, the sojourn density of $R_{\infty}^{*}\left(T_{\infty}(t)\right)$ with respect to $2^{-1} d m(x)$ is the same as that of $R_{\infty}(t)$ with respect to $d x$, and in going from one to the other the change of time scale from $t$ tc $T_{\infty}(t)$ cannot be avoided.

As an application of these facts, we consider the radial component $Y(t)$ of a Brownian motion in $N>1$ dimensions. This is known to be a diffusion process for which

$$
P\{Y(t) \leqq r\}=\frac{2}{2^{N} \Gamma\left(\frac{1}{2} N\right)} \int_{0}^{r \sqrt{ } t} \rho^{N-1} e^{-\rho^{2} / 2} d \rho
$$


holds. Using this and the spacial homogeneity of Brownian motion, it can be shown that the generator of this process is $\frac{1}{2}\left(\partial^{2} / \partial r^{2}\right)+((N-1) / 2 r) i(\partial / \partial r)$. With this generator as starting point, we cannot apply the preceding analysis directly because of the presence of a "drift" in the positive direction, implying that the generator does not have the form $(d / d m)(d / d x)$. However, if a change of scale is introduced by the transformation $z=f(r)$, where $f$ is a solution of $\left(\frac{1}{2}\left(\partial^{2} / \partial r^{2}\right)+((N-1) / 2 r)(\partial / \partial r)\right) f=0$, it is known that the generator assumes the required form. We shall consider only the case $N>2$; the case $N=2$ may be treated similarly but requires special formulas. For $N>2$ the necessary change of scale is $z=(N-2)^{-1} r^{-(N-2)}$, in terms of which if the corresponding process is written $Z(t)=z(Y(t))$, then the generator of $Z(t)$ assumes the form $(d / d m)(d / d z)$ with

$$
m(z)=2(N-2)^{-2-2 /(N-2)} \int_{r_{0}}^{z} x^{-2-2 /(N-2)} d x .
$$

We consider for the moment a change of starting point letting $Y(0)=r_{0}>0$. The total sojourn time period of $Y(t)$ in $\left(0, r_{1}\right)$ before reaching $r_{2}$, for $r_{0} \leqq r_{1} \leqq r_{2}$, is the same as that of $Z(t)$ in $\left(r_{1}^{-(N-2)} /(N-2), \infty\right)$, which in view of the above considerations can be written in the form

$$
8) \int_{\alpha}^{\infty} X(x) d m\left(x+\frac{r_{2}^{-(N-2)}}{N-2}\right)=\int_{\alpha}^{\infty}\left(2\left((N-2) x+r_{2}^{-(N-2)}\right)-2-2 /(N-2) X(x) d x\right.
$$

where $\alpha=\left(r_{1}^{-(N-2)}-r_{2}^{-(N-2)}\right) /(N-2)$ and $X(x)$ is the process of Corollary 1.3 with $-x_{0}=\left(r_{0}^{-(N-2)}-{\overline{r_{2}}}^{-(N-2)}\right) /(N-2)$ and $\alpha_{0}=0$. Letting $r_{0}$ approach 0 and $r_{2}$ approach $\infty$, the total sojourn time period of $Y(t)$ starting at 0 in $\left(0, r_{1}\right)$ becomes

$$
\int_{(N-2)^{-1} r_{1}-(N-2)}^{\infty} 2((N-2) x)^{-2-2 /(N-2)} X(x) d x,
$$

where $X(x)$ is the diffusion process with initial value 0 and generator $z d^{2} / d z^{2}+d / d z$. The new feature of this representation is that it presents the total sojourn time as a stochastic process with parameter $r_{1}$. For $r_{1}$ fixed, the distribution of this random quantity is completely given in [1]. Let us remark, finally, that in [1] it is shown that for $N>2$ the distribution of the total sojourn time in a sphere of radius $r_{1}$ is identical with that of the first passage time to the sphere of radius $r_{1}$ in dimension $N-2$. It is to be noted here that this first passage time may be represented for $N>4$ by letting $r_{2}=r_{1}$ and $r_{0}=0$ in (4.8), which gives the expression

$$
\int_{0}^{\infty} 2\left((N-4)\left(x+r_{1}^{-(N-4)}(N-4)^{-1}\right)\right)^{-2-2 /(N-4)} X(x) d x .
$$


Using the easily verified facts that $E(X(x))=x$ and $E\left(X\left(x_{1}\right) X\left(x_{2}\right)\right)=x_{1}\left(x_{2}+x_{1}\right)$ it can be shown directly that the first two moments of the distributions of (4.9) and (4.10) are the same. To verify directly the identity of the two distributions, however, remains an open problem.

\section{REFERENCES}

1. Z. Ciesielski and S. J. Taylor, First passage times and sojourn times for Brownian motion in space and exact Hausdorff measure of the sample path, Trans. Amer. Math. Soc. 103 (1962), 434-450.

2. H. Cramer, Sur un nouveau théorème-limite de la thíorie des probabilités, Actualités Sci. Ind. 736 (1938).

3. D. A. Darling and M. Kac, On occupation times for Markov processes, Trans. Amer. Math. Soc. 84 (1957), 444-458.

4. E. B. Dynkin, Markov processes and semigroups of operators, Theor. Probability Appl. 1 (1956), 22-23.

5. W. Feller, Die Grundlagen der Volterraschen Theorie des Kampfes ums Dasein in wahrscheinlichkeitstheoretischer Behandlung, Acta Biotheor. 5 (1939), 11-40.

6. - On the intrinsic form for second order differential operators, Illinois J. Math. 2 (1958), 1-18.

7. —, An introduction to probability theory and its applications, 2nd ed., Vol. 1, Wiley, New York, 1957.

8. K. Ito and H. P. McKean, Monograph on difflision, Springer, Berlin, 1963.

9. M. Kac, On some connections between probability and differential and integral equations, Proc. 2nd Berkeley Symposium on Math. Statist. and Prob., pp. 189-215, Univ. of California Press, Berkeley, Calif., 1951.

10. F. B. Knight, On the random walk and Brownian motion, Trans. Amer. Math. Soc. 103 (1962), 218-228.

11. P. Lévy, Processus stochastique et mouvement Brownien, Gauthier-Villars, Paris, 1948.

12. - Sur certains processus stochastiques homogènes, Compositio Math. 7 (1939), 283-339.

13. D. Ray, Sojourn times of diffusion processes, Illinois J. Math. (1963) (to appear).

14. H. Trotter, A property of Brownian motion paths, Illinois J. Math. 2 (1958), 425-433.

UNIVERSITY OF MINNESOTA, MinNeA polis, MinNesota. 